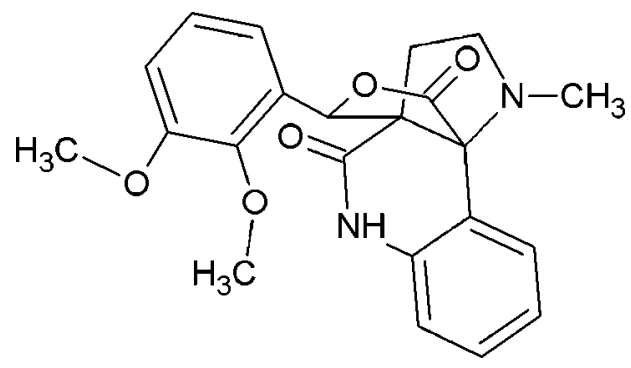

\title{
Crystal structure of 11-(2,3-dimethoxy-
} phenyl)-14-methyl-12-oxa-8,14-diazatetracyclo $\left[8.3 \cdot 3 \cdot 0^{1,10} \cdot 0^{2,7}\right]$ hexadeca2(7),3,5-triene-9,13-dione

\author{
M. P. Savithri, ${ }^{a}$ M. Suresh, ${ }^{b}$ R. Raghunathan, ${ }^{b}$ R. Raja ${ }^{c}$ and \\ A. SubbiahPandi ${ }^{\mathrm{C} *}$ \\ a Department of Physics, Queen Mary's College (Autonomous), Chennai 600 004, \\ India, 'bepartment of Organic Chemistry, University of Madras, Guindy Campus, \\ Chennai 600 025, India, and ' Department of Physics, Presidency College \\ (Autonomous), Chennai 600005 , India. *Correspondence e-mail: \\ aspandian59@gmail.com
}

Received 23 March 2015; accepted 29 March 2015

Edited by W. T. A. Harrison, University of Aberdeen, Scotland

The title compound, $\mathrm{C}_{22} \mathrm{H}_{22} \mathrm{~N}_{2} \mathrm{O}_{5}$, contains two conformationally similar molecules $(A$ and $B)$ in its the asymmetric unit (r.m.s. overlay fit for the 29 non-H atoms $=0.194 \AA$ ). In each molecule, the lactone ring has an envelope conformation with the spiro $\mathrm{C}$ atom as the flap. In the crystal, $A+A$ and $B+B$ inversion dimers linked by pairs of $\mathrm{N}-\mathrm{H} \cdots \mathrm{O}$ hydrgen bonds occur; in both cases, $R_{2}^{2}(8)$ loops are generated. A weak $\mathrm{C}-$ $\mathrm{H}$.. O interaction is also observed, which links the dimers into [010] chains.

Keywords: crystal structure; diazatetracyclhexadecatrienedione; $\mathrm{N}-$ $\mathrm{H}$... O hydrgen bonds.

CCDC reference: 1056691

\section{Related literature}

For general background and the biological and pharmacological properties of quinoline derivatives, see: Michael (1997). For a related structure, see: Vennila et al. (2011).

\section{Experimental}

\subsection{Crystal data}

$\mathrm{C}_{22} \mathrm{H}_{22} \mathrm{~N}_{2} \mathrm{O}_{5}$

$M_{r}=394.42$

Triclinic, $P \overline{1}$

$a=10.1360$ (4) $\AA$

$b=10.3198(4) \AA$

$c=18.8973(7) \AA$

$\alpha=89.079(2)^{\circ}$

$\beta=74.955(2)^{\circ}$

\subsection{Data collection}

Bruker Kappa APEXII CCD diffractometer

Absorption correction: multi-scan (SADABS; Bruker, 2004)

$T_{\min }=0.967, T_{\max }=0.971$

\subsection{Refinement}

$R\left[F^{2}>2 \sigma\left(F^{2}\right)\right]=0.047$

$w R\left(F^{2}\right)=0.128$

$S=1.02$

6717 reflections

532 parameters

Table 1

Hydrogen-bond geometry $\left(\AA{ }^{\circ}\right)$.

\begin{tabular}{|c|c|c|c|c|}
\hline$D-\mathrm{H} \cdots A$ & $D-\mathrm{H}$ & $\mathrm{H} \cdots A$ & $D \cdots A$ & $D-\mathrm{H} \cdots A$ \\
\hline $\mathrm{N} 8-\mathrm{H} 8 \cdots \mathrm{O} 9^{\mathrm{i}}$ & $0.89(3)$ & $2.01(3)$ & $2.903(2)$ & $177(2)$ \\
\hline $\mathrm{N} 8 A-\mathrm{H} 8 A \cdots \mathrm{O} 9 A^{\mathrm{ii}}$ & $0.89(2)$ & $2.07(3)$ & $2.958(2)$ & $175(2)$ \\
\hline $\mathrm{C} 6 A^{\prime}-\mathrm{H} 6 A^{\prime} \cdots \mathrm{O} 13 A^{\mathrm{iii}}$ & 0.93 & 2.43 & $3.322(3)$ & 161 \\
\hline
\end{tabular}

Data collection: APEX2 (Bruker, 2004); cell refinement: SAINT (Bruker, 2004); data reduction: $S A I N T$; program(s) used to solve structure: SHELXS97 (Sheldrick, 2008); program(s) used to refine structure: SHELXL97 (Sheldrick, 2008); molecular graphics: ORTEP-3 for Windows (Farrugia, 2012); software used to prepare material for publication: SHELXL97 and PLATON (Spek, 2009).

\section{Acknowledgements}

The authors thank Dr Babu Vargheese, SAIF, IIT, Madras, India, for the data collection. 


\section{data reports}

Supporting information for this paper is available from the IUCr electronic archives (Reference: HB7391).

\section{References}

Bruker (2004). APEX2, SAINT and $S A D A B S$. Bruker AXS Inc, Madison, Wisconsin, USA.
Farrugia, L. J. (2012). J. Appl. Cryst. 45, 849-854.

Michael, J. P. (1997). Nat. Prod. Rep. 14, 605-608.

Sheldrick, G. M. (2008). Acta Cryst. A64, 112-122.

Spek, A. L. (2009). Acta Cryst. D65, 148-155.

Vennila, K. N., Sankaran, M., Mohan, P. S. \& Velmurugan, D. (2011). Acta Cryst. E67, o3376-o3377. 


\title{
supporting information
}

Acta Cryst. (2015). E71, o293-o294 [https://doi.org/10.1107/S2056989015006386]

\section{Crystal structure of 11-(2,3-dimethoxyphenyl)-14-methyl-12-oxa-8,14-diaza- tetracyclo $\left[8.3 .3 .0^{1,10} \cdot 0^{2,7}\right]$ hexadeca-2(7),3,5-triene-9,13-dione}

\author{
M. P. Savithri, M. Suresh, R. Raghunathan, R. Raja and A. SubbiahPandi
}

\section{S1. Comment}

Quinolines exhibit physico-chemical activities which are useful in the field of pharmaceuticals and agrochemicals. Their derivatives are also present in a wide variety of natural products involved in several biological activities (Michael, 2006). The crystal structure of the title compound is presented here as a part of our on-going structural studies on quinoline derivatives.

The molecular structure of molecule (A) and molecule (B) is shown in Fig.1. The furan ring (O12A/C11A$\mathrm{C} 10 \mathrm{~A} / \mathrm{C} 1 \mathrm{~A} / \mathrm{C} 13 \mathrm{~A})$ of $(\mathrm{A})$ exhibits an envelope conformation with $\mathrm{C} 10 \mathrm{~A}$ as the flap atom. The furan ring $(\mathrm{O} 12 / \mathrm{C} 11-$ $\mathrm{C} 10 / \mathrm{C} 1 / \mathrm{C} 13)$ of $(\mathrm{B})$ exhibits an envelope conformation with $\mathrm{C} 10$ as the flap atom. The quinoline ring $(\mathrm{N} 8 \mathrm{~A} / \mathrm{C} 1 \mathrm{~A}-\mathrm{C} 10 \mathrm{~A})$ is almost coplanar showing a dihedral angle of $2.9(8)^{\circ}$ with the pyridine ring $(\mathrm{N} 8 \mathrm{~A} / \mathrm{C} 7 \mathrm{~A} / \mathrm{C} 2 \mathrm{~A}-\mathrm{C} 1 \mathrm{~A} / \mathrm{C} 9 \mathrm{~A}-\mathrm{C} 10 \mathrm{~A})$ of molecule (A) and is perpendicular with the pyridine ring (N8/C7/C2-C1/C9-C10) of molecule (B) inclined at an angle of $88.3(8)^{\circ}$. The sum of angles at N8, N8A of the quinoline rings $\left(360^{\circ}\right)$ is in accordance with $\mathrm{sp}^{2}$ hybridization.

In the crystal of two independent molecules, hydrogen-bonded chains running along bc plane are generated by connecting neighbouring molecules via $\mathrm{N}-\mathrm{H} \cdots \mathrm{O}, \mathrm{C}-\mathrm{H} \cdots \mathrm{O}$ hydrogen bonds forming a two dimensional structure (Fig.2.) The hydrogen bonds of N8-H8 $\cdots \mathrm{O} 9$ and N8A-H8A $\cdots \mathrm{O} 9 \mathrm{~A}$ forming inversion dimers but enclosing smaller $\mathrm{R}_{2}^{2}(8)$ loops and the hydrogen bond of $\mathrm{C}^{2} \mathrm{~A}^{\prime}-\mathrm{H}_{6} \mathrm{~A}^{\prime} \cdots \mathrm{O} 13 \mathrm{~A}$ forms a one dimensional chain along [010] as shown in Fig.3.

\section{S2. Experimental}

A mixture of methyl 2-(hydroxy(m-tolyl)methyl)acrylate (252mgs, $1 \mathrm{mmol})$, isatin (161.7mgs, $1.1 \mathrm{mmol})$ and sarcosine $(97.9 \mathrm{mgs}, 1.1 \mathrm{mmol})$ was placed in a round bottom flask and melted at $180^{\circ} \mathrm{C}$ until completion of the reaction was evidenced by TLC analysis. After completion of the reaction, the crude product was washed with $5 \mathrm{ml}$ of ethylacetate and hexane mixture (1:4 ratio) which successfully provided the pure product as colorless solid. The product was dissolved in ethyl acetate and heated for two minutes. The resulting solution was subjected to crystallization by slow evaporation of the solvent for 48 hours resulting in the formation of colourless blocks.

\section{S3. Refinement}

All $\mathrm{H}$ atoms were fixed geometrically and allowed to ride on their parent $\mathrm{C}$ atoms, with $\mathrm{C}-\mathrm{H}$ distances fixed in the range 0.93-0.98 $\AA$ with $U_{\text {iso }}(\mathrm{H})=1.5 U_{\mathrm{eq}}(\mathrm{C})$ for methyl $\mathrm{H} 1.2 U_{\mathrm{eq}}(\mathrm{C})$ for other $\mathrm{H}$ atoms. 


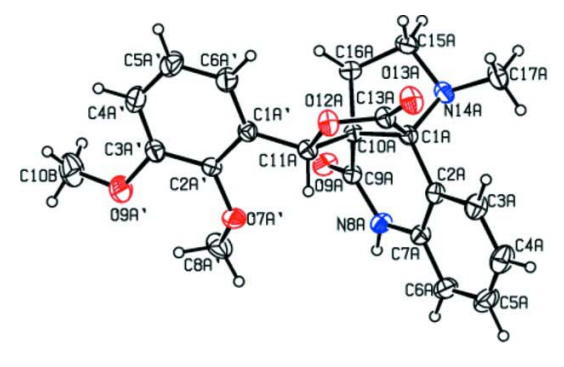

MOLECLUE-A

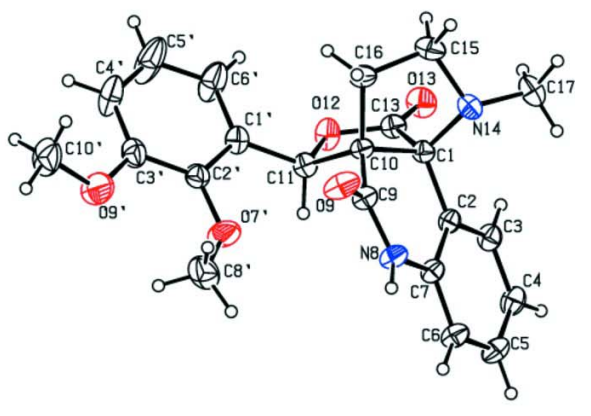

MOLECULE-B

\section{Figure 1}

The molecular structure of the title compound with the atom-numbering scheme. Displacement ellipsoids are drawn at the $30 \%$ probability level. $\mathrm{H}$ atoms are omitted for clarity. 


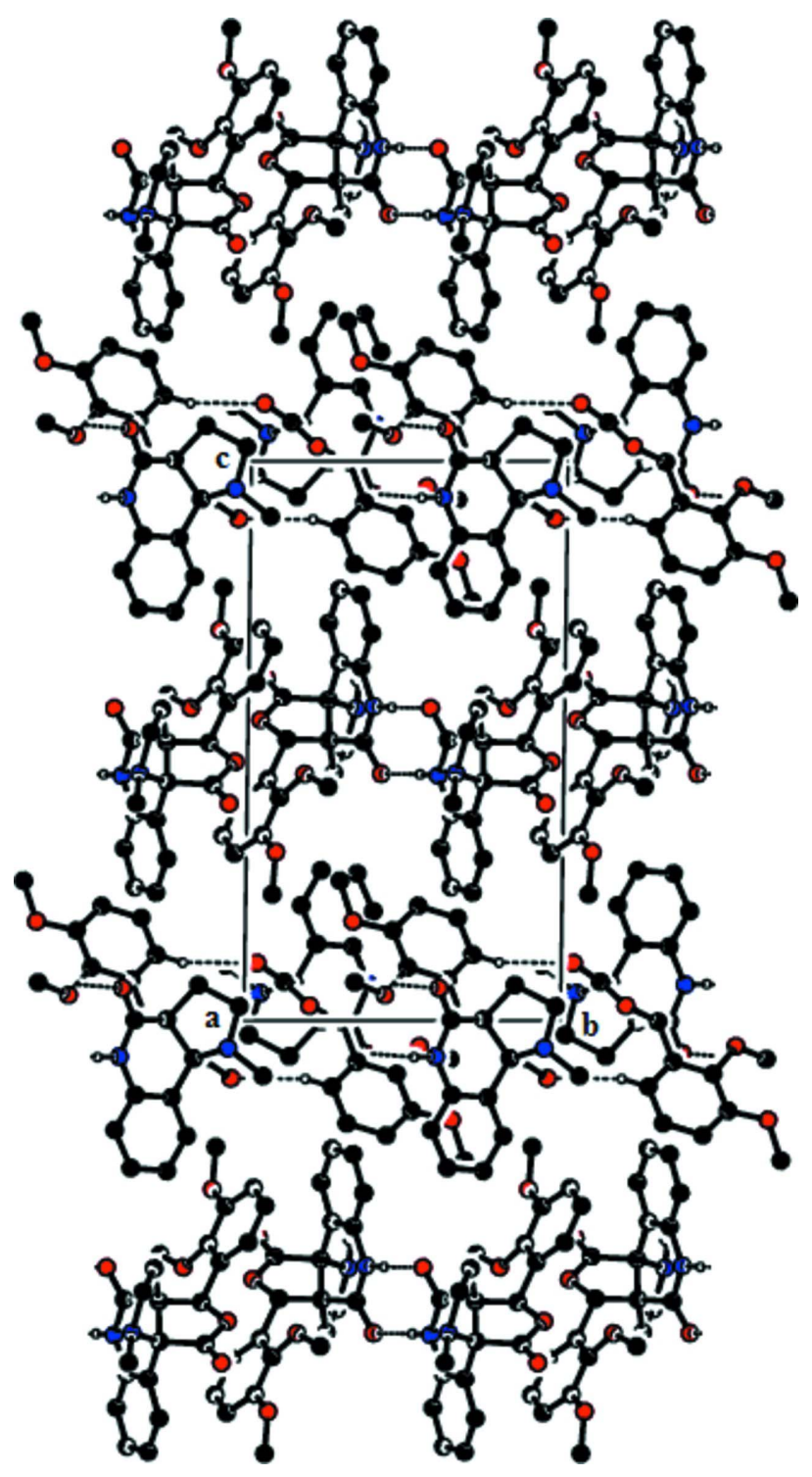

Figure 2

The molecular packing is viewed along the $a$ axis. Dashed lines show the intermolecular $\mathrm{N}-\mathrm{H} \cdots \mathrm{O}$ and $\mathrm{C}-\mathrm{H} \cdots \mathrm{O}$ hydrogen bonds. $\mathrm{H}$ atoms not involved in hydrogen bonding have been omitted for clarity. 


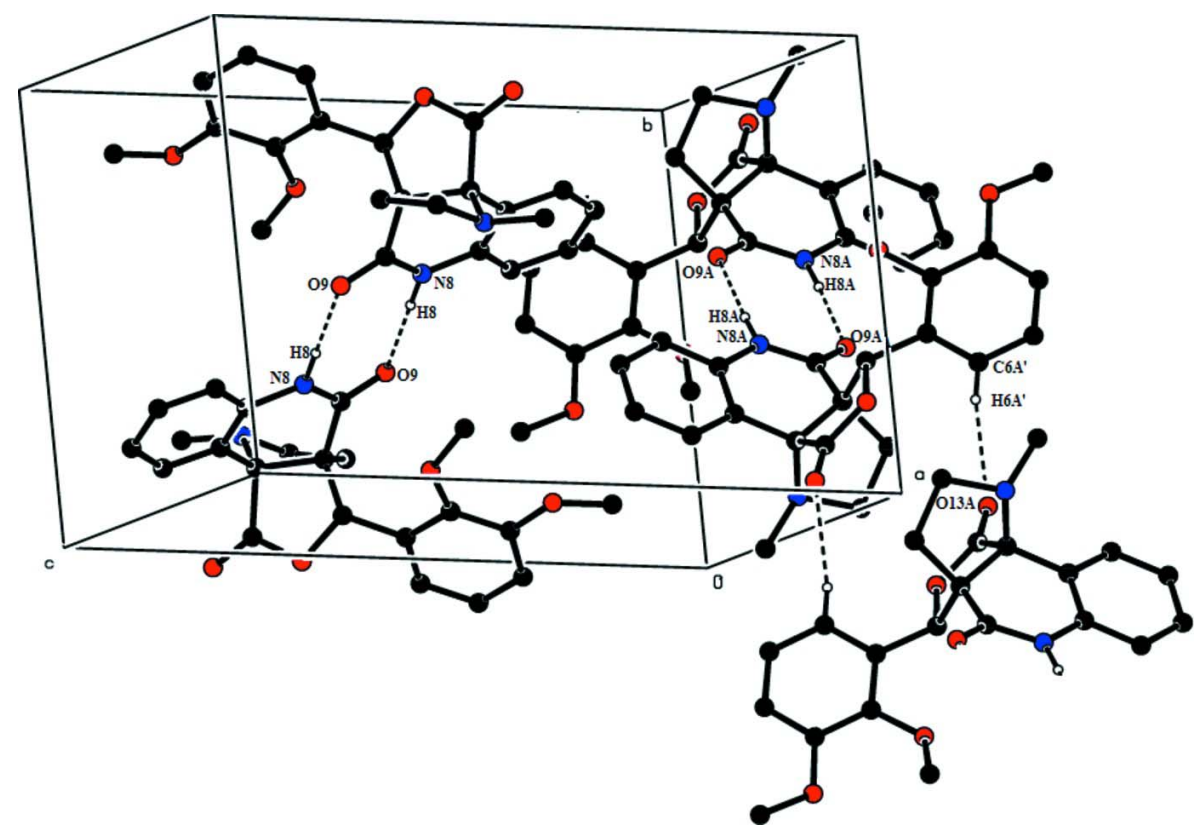

Figure 3

A partial view of the $\mathrm{N} 8-\mathrm{H} 8 \cdots \mathrm{O} 9, \mathrm{~N} 8 A-\mathrm{H} 8 A^{\cdots} \cdots \mathrm{O} 9 A$ and $\mathrm{C} 6 A^{\prime}-\mathrm{H} 6 A^{\prime} \cdots \mathrm{O} 13 A$ hydrogen-bonding interactions along the $c$ axis.

11-(2,3-Dimethoxyphenyl)-14-methyl-12-oxa-8,14-diazatetracyclo[8.3.3.0 $\left.{ }^{1,10} \cdot 0^{2,7}\right]$ hexadeca-2(7),3,5-triene-9,13dione

Crystal data

$\mathrm{C}_{22} \mathrm{H}_{22} \mathrm{~N}_{2} \mathrm{O}_{5}$

$M_{r}=394.42$

Triclinic, $P \overline{1}$

Hall symbol: -P 1

$a=10.1360$ (4) $\AA$

$b=10.3198(4) \AA$

$c=18.8973(7) \AA$

$\alpha=89.079(2)^{\circ}$

$\beta=74.955(2)^{\circ}$

$\gamma=89.406(2)^{\circ}$

$V=1908.64(13) \AA^{3}$

\section{Data collection}

Bruker Kappa APEXII CCD diffractometer

Radiation source: fine-focus sealed tube Graphite monochromator $\omega$ and $\varphi$ scans

Absorption correction: multi-scan

(SADABS; Bruker, 2004)

$T_{\min }=0.967, T_{\max }=0.971$
$Z=4$

$F(000)=832$

$D_{\mathrm{x}}=1.373 \mathrm{Mg} \mathrm{m}^{-3}$

Mo $K \alpha$ radiation, $\lambda=0.71073 \AA$

Cell parameters from 6717 reflections

$\theta=1.1-25.0^{\circ}$

$\mu=0.10 \mathrm{~mm}^{-1}$

$T=293 \mathrm{~K}$

Block, colourless

$0.35 \times 0.30 \times 0.30 \mathrm{~mm}$

35145 measured reflections

6717 independent reflections

5408 reflections with $I>2 \sigma(I)$

$R_{\text {int }}=0.028$

$\theta_{\text {max }}=25.0^{\circ}, \theta_{\text {min }}=1.1^{\circ}$

$h=-12 \rightarrow 12$

$k=-12 \rightarrow 12$

$l=-22 \rightarrow 22$ 


\section{Refinement}

Refinement on $F^{2}$

Least-squares matrix: full

$R\left[F^{2}>2 \sigma\left(F^{2}\right)\right]=0.047$

$w R\left(F^{2}\right)=0.128$

$S=1.02$

6717 reflections

532 parameters

0 restraints

Primary atom site location: structure-invariant direct methods

Secondary atom site location: difference Fourier map
Hydrogen site location: inferred from neighbouring sites

$\mathrm{H}$ atoms treated by a mixture of independent and constrained refinement

$w=1 /\left[\sigma^{2}\left(F_{\mathrm{o}}^{2}\right)+(0.0555 P)^{2}+1.1105 P\right]$ where $P=\left(F_{\mathrm{o}}{ }^{2}+2 F_{\mathrm{c}}{ }^{2}\right) / 3$

$(\Delta / \sigma)_{\max }<0.001$

$\Delta \rho_{\max }=0.65$ e $\AA^{-3}$

$\Delta \rho_{\min }=-0.54$ e $\AA^{-3}$

Extinction correction: SHELXL97 (Sheldrick, 2008), $\mathrm{Fc}^{*}=\mathrm{kFc}\left[1+0.001 \times \mathrm{xc}^{2} \lambda^{3} / \sin (2 \theta)\right]^{-1 / 4}$

Extinction coefficient: 0.0073 (9)

\section{Special details}

Geometry. All esds (except the esd in the dihedral angle between two 1.s. planes) are estimated using the full covariance matrix. The cell esds are taken into account individually in the estimation of esds in distances, angles and torsion angles; correlations between esds in cell parameters are only used when they are defined by crystal symmetry. An approximate (isotropic) treatment of cell esds is used for estimating esds involving l.s. planes.

Refinement. Refinement of $\mathrm{F}^{2}$ against ALL reflections. The weighted R-factor $\mathrm{wR}$ and goodness of fit $\mathrm{S}$ are based on $\mathrm{F}^{2}$, conventional $\mathrm{R}$-factors $\mathrm{R}$ are based on $\mathrm{F}$, with $\mathrm{F}$ set to zero for negative $\mathrm{F}^{2}$. The threshold expression of $\mathrm{F}^{2}>2 \operatorname{sigma}\left(\mathrm{F}^{2}\right)$ is used only for calculating R-factors(gt) etc. and is not relevant to the choice of reflections for refinement. R-factors based on $\mathrm{F}^{2}$ are statistically about twice as large as those based on F, and R- factors based on ALL data will be even larger.

Fractional atomic coordinates and isotropic or equivalent isotropic displacement parameters $\left(\AA^{2}\right)$

\begin{tabular}{lllll}
\hline & $x$ & $y$ & $z$ & $U_{\text {iso }} / U_{\mathrm{eq}}$ \\
\hline C10B & $1.0615(3)$ & $0.7030(3)$ & $-0.25020(16)$ & $0.0810(9)$ \\
H10A & 1.0438 & 0.7943 & -0.2528 & $0.121^{*}$ \\
H10B & 1.0127 & 0.6574 & -0.2792 & $0.121^{*}$ \\
H10C & 1.1577 & 0.6868 & -0.2686 & $0.121^{*}$ \\
H8A & $0.517(2)$ & $0.461(2)$ & $0.0654(12)$ & $0.049(6)^{*}$ \\
H8 & $0.057(3)$ & $0.546(2)$ & $0.4402(13)$ & $0.061(7)^{*}$ \\
C1A & $0.71903(18)$ & $0.15796(17)$ & $0.06757(10)$ & $0.0323(4)$ \\
O12A & $0.95534(13)$ & $0.20658(13)$ & $0.02653(8)$ & $0.0433(4)$ \\
O9A & $0.61164(15)$ & $0.38520(14)$ & $-0.05792(7)$ & $0.0448(4)$ \\
N8A & $0.57191(17)$ & $0.39272(17)$ & $0.06429(9)$ & $0.0379(4)$ \\
C9A & $0.63693(18)$ & $0.34543(18)$ & $-0.00142(10)$ & $0.0324(4)$ \\
O13A & $0.90527(15)$ & $0.03882(14)$ & $0.10304(9)$ & $0.0530(4)$ \\
O7A & $0.86006(16)$ & $0.55593(14)$ & $-0.04580(8)$ & $0.0513(4)$ \\
C11A & $0.88282(18)$ & $0.30883(18)$ & $-0.00169(10)$ & $0.0337(4)$ \\
H11A & 0.8642 & 0.3801 & 0.0333 & $0.040^{*}$ \\
C7A & $0.5767(2)$ & $0.34058(19)$ & $0.13261(10)$ & $0.0367(4)$ \\
C10A & $0.74578(18)$ & $0.24380(17)$ & $-0.00209(10)$ & $0.0301(4)$ \\
N14A & $0.64191(16)$ & $0.04987(15)$ & $0.04930(9)$ & $0.0389(4)$ \\
C1A' & $0.97041(19)$ & $0.35816(18)$ & $-0.07344(11)$ & $0.0363(4)$ \\
C2A & $0.64674(19)$ & $0.22556(19)$ & $0.13708(10)$ & $0.0355(4)$ \\
C16A & $0.7560(2)$ & $0.14502(19)$ & $-0.06377(11)$ & $0.0400(5)$ \\
H16A & 0.6961 & 0.1700 & -0.0943 & $0.048^{*}$ \\
H16B & 0.8489 & 0.1397 & -0.0943 & $0.048^{*}$ \\
& & & &
\end{tabular}




\begin{tabular}{|c|c|c|c|c|}
\hline $\mathrm{C} 13 \mathrm{~A}$ & $0.8671(2)$ & 0.12459 (19) & $0.06982(11)$ & $0.0378(5)$ \\
\hline $\mathrm{C} 2 \mathrm{~A}^{\prime}$ & $0.9520(2)$ & $0.48460(18)$ & $-0.09543(11)$ & $0.0362(4)$ \\
\hline $\mathrm{C} 3 \mathrm{~A}^{\prime}$ & $1.0346(2)$ & $0.5333(2)$ & $-0.16135(12)$ & $0.0457(5)$ \\
\hline $\mathrm{C} 3 \mathrm{~A}$ & $0.6440(2)$ & $0.1761(2)$ & $0.20631(12)$ & $0.0504(6)$ \\
\hline $\mathrm{H} 3 \mathrm{~A}$ & 0.6921 & 0.1004 & 0.2105 & $0.060 *$ \\
\hline $\mathrm{C} 15 \mathrm{~A}$ & $0.7124(2)$ & $0.0159(2)$ & $-0.02557(12)$ & $0.0449(5)$ \\
\hline H15A & 0.6518 & -0.0293 & -0.0489 & $0.054 *$ \\
\hline H15B & 0.7912 & -0.0388 & -0.0265 & $0.054^{*}$ \\
\hline C6A & $0.5070(2)$ & $0.4038(2)$ & $0.19557(12)$ & $0.0512(6)$ \\
\hline H6A & 0.4620 & 0.4818 & 0.1921 & $0.061^{*}$ \\
\hline O9A' & $1.0186(2)$ & $0.66034(16)$ & $-0.17763(10)$ & $0.0789(6)$ \\
\hline C6 $A^{\prime}$ & $1.0697(2)$ & $0.2811(2)$ & $-0.11824(14)$ & $0.0544(6)$ \\
\hline $\mathrm{H}_{6} \mathrm{~A}^{\prime}$ & 1.0829 & 0.1963 & -0.1039 & $0.065^{*}$ \\
\hline $\mathrm{C} 4 \mathrm{~A}^{\prime}$ & $1.1314(3)$ & $0.4546(2)$ & $-0.20506(14)$ & $0.0599(6)$ \\
\hline $\mathrm{H} 4 \mathrm{~A}^{\prime}$ & 1.1855 & 0.4864 & -0.2493 & $0.072 *$ \\
\hline $\mathrm{C} 4 \mathrm{~A}$ & $0.5714(3)$ & $0.2371(3)$ & $0.26883(13)$ & $0.0615(7)$ \\
\hline $\mathrm{H} 4 \mathrm{~A}$ & 0.5680 & 0.2010 & 0.3147 & $0.074 *$ \\
\hline C17A & $0.6138(2)$ & $-0.0621(2)$ & $0.09891(14)$ & $0.0548(6)$ \\
\hline H17A & 0.5677 & -0.0341 & 0.1473 & $0.082 *$ \\
\hline H17B & 0.6983 & -0.1040 & 0.1000 & $0.082 *$ \\
\hline $\mathrm{H} 17 \mathrm{C}$ & 0.5571 & -0.1219 & 0.0821 & $0.082 *$ \\
\hline $\mathrm{C} 5 \mathrm{~A}$ & $0.5043(3)$ & $0.3513(3)$ & $0.26324(13)$ & $0.0610(7)$ \\
\hline $\mathrm{H} 5 \mathrm{~A}$ & 0.4568 & 0.3934 & 0.3054 & $0.073 *$ \\
\hline $\mathrm{C} 8 \mathrm{~A}^{\prime}$ & $0.7778(3)$ & $0.6521(2)$ & $-0.06863(16)$ & $0.0621(7)$ \\
\hline H8A1 & 0.7195 & 0.6925 & -0.0264 & $0.093 *$ \\
\hline $\mathrm{H} 8 \mathrm{~A} 2$ & 0.7228 & 0.6124 & -0.0966 & $0.093 *$ \\
\hline H8A3 & 0.8354 & 0.7163 & -0.0984 & $0.093 *$ \\
\hline $\mathrm{C} 5 \mathrm{~A}^{\prime}$ & $1.1486(3)$ & $0.3291(3)$ & $-0.18348(15)$ & $0.0672(7)$ \\
\hline $\mathrm{H} 5 \mathrm{~A}^{\prime}$ & 1.2143 & 0.2764 & -0.2133 & $0.081^{*}$ \\
\hline N8 & $0.12270(18)$ & $0.60382(17)$ & $0.43819(9)$ & $0.0413(4)$ \\
\hline $\mathrm{O} 12$ & $0.29646(15)$ & $0.96066(13)$ & $0.46254(8)$ & $0.0480(4)$ \\
\hline $\mathrm{C} 1$ & $0.36492(18)$ & $0.74700(18)$ & $0.42665(10)$ & $0.0334(4)$ \\
\hline $\mathrm{C} 2$ & $0.31784(19)$ & $0.71338(18)$ & $0.35954(10)$ & 0.0348 \\
\hline $\mathrm{C} 7$ & $0.1971(2)$ & $0.64514(18)$ & $0.36828(10)$ & $0.0360(4)$ \\
\hline O9 & $0.09671(17)$ & $0.57648(16)$ & $0.55871(8)$ & $0.0579(5)$ \\
\hline $\mathrm{O} 13$ & $0.50407(15)$ & $0.94355(15)$ & $0.38830(9)$ & $0.0551(4)$ \\
\hline $\mathrm{C} 10$ & $0.25101(19)$ & $0.74324(18)$ & $0.49834(10)$ & $0.0322(4)$ \\
\hline $\mathrm{C} 11$ & $0.1820(2)$ & $0.87718(18)$ & $0.49699(11)$ & $0.0374(4)$ \\
\hline H11 & 0.1197 & 0.8733 & 0.4652 & $0.045^{*}$ \\
\hline N14 & $0.46780(16)$ & $0.65966(17)$ & $0.44204(10)$ & 0.0423 \\
\hline C9 & $0.1510(2)$ & $0.63346(19)$ & $0.50162(10)$ & $0.0372(4)$ \\
\hline $\mathrm{C} 13$ & $0.4009(2)$ & 0.89217 (19) & $0.42184(11)$ & $0.0388(5)$ \\
\hline $\mathrm{C} 1^{\prime}$ & $0.1075(2)$ & $0.93454(19)$ & $0.56880(12)$ & $0.0426(5)$ \\
\hline $\mathrm{C} 3$ & $0.3898(2)$ & $0.7494(2)$ & $0.28913(11)$ & $0.0426(5)$ \\
\hline $\mathrm{H} 3$ & 0.4697 & 0.7969 & 0.2823 & $0.051^{*}$ \\
\hline $\mathrm{C} 4$ & $0.3449(2)$ & $0.7160(2)$ & $0.22934(11)$ & $0.0471(5)$ \\
\hline $\mathrm{H} 4$ & 0.3957 & 0.7387 & 0.1824 & $0.057^{*}$ \\
\hline $\mathrm{C} 2^{\prime}$ & $-0.0258(2)$ & $0.8978(2)$ & $0.60066(11)$ & $0.0432(5)$ \\
\hline
\end{tabular}




$\begin{array}{lllll}\text { C16 } & 0.3306(2) & 0.7269(2) & 0.55711(11) & 0.0437(5) \\ \text { H16C } & 0.3282 & 0.8066 & 0.5840 & 0.052^{*} \\ \text { H16D } & 0.2918 & 0.6580 & 0.5914 & 0.052^{*} \\ \text { C6 } & 0.1502(2) & 0.6138(2) & 0.30803(11) & 0.0461(5) \\ \text { H6 } & 0.0687 & 0.5691 & 0.3145 & 0.055^{*} \\ \text { C5 } & 0.2246(2) & 0.6489(2) & 0.23883(11) & 0.0491(5) \\ \text { H5 } & 0.1937 & 0.6273 & 0.1983 & 0.059^{*} \\ \text { O7' } & -0.0777(17) & 0.8186(2) & 0.55922(10) & 0.0873(7) \\ \text { C15 } & 0.4763(2) & 0.6935(2) & 0.51562(12) & 0.0511(6) \\ \text { H15C } & 0.5360 & 0.7671 & 0.5137 & 0.061^{*} \\ \text { H15D } & 0.5109 & 0.6209 & 0.5389 & 0.061^{*} \\ \text { C3' } & -0.0965(2) & 0.9489(2) & 0.66811(13) & 0.0518(6) \\ \text { C6' } & 0.1686(3) & 1.0237(3) & 0.60371(16) & 0.0825(10) \\ \text { H6' } & 0.2582 & 1.0491 & 0.5828 & 0.099^{*} \\ \text { O9' } & -0.22558(19) & 0.9071(3) & 0.69852(11) & 0.0991(8) \\ \text { C17 } & 0.5998(2) & 0.6501(2) & 0.38864(14) & 0.0579(6) \\ \text { H17D } & 0.6568 & 0.5892 & 0.4061 & 0.087^{*} \\ \text { H17E } & 0.6426 & 0.7335 & 0.3817 & 0.087^{*} \\ \text { H17F } & 0.5871 & 0.6212 & 0.3428 & 0.087^{*} \\ \text { C5' } & 0.0973(4) & 1.0747(3) & 0.66906(19) & 0.1108(15) \\ \text { H5' } & 0.1388 & 1.1352 & 0.6920 & 0.133^{*} \\ \text { C4' } & -0.0340(3) & 1.0379(3) & 0.70101(16) & 0.0753(9) \\ \text { H4' } & -0.0811 & 1.0736 & 0.7454 & 0.090^{*} \\ \text { C8' } & -0.1935(3) & 0.7448(3) & 0.58216(18) & 0.0735(8) \\ \text { H8'1 } & -0.2080 & 0.6977 & 0.5415 & 0.110^{*} \\ \text { H8'2 } & -0.2707 & 0.8003 & 0.6013 & 0.110^{*} \\ \text { H8'3 } & -0.1827 & 0.6849 & 0.6197 & 0.110^{*} \\ \text { C10' } & -0.2794(3) & 0.9163(3) & 0.77410(16) & 0.0827(9) \\ \text { H10D } & -0.3707 & 0.8829 & 0.7874 & 0.124^{*} \\ \text { H10E } & -0.2809 & 1.0055 & 0.7881 & 0.124^{*} \\ \text { H10F } & -0.2236 & 0.8670 & 0.7988 & 0.124^{*} \\ & & & & \end{array}$

Atomic displacement parameters $\left(\AA^{2}\right)$

\begin{tabular}{lllllll}
\hline & $U^{11}$ & $U^{22}$ & $U^{33}$ & $U^{12}$ & $U^{13}$ & $U^{23}$ \\
\hline C10B & $0.101(2)$ & $0.0716(19)$ & $0.0727(19)$ & $-0.0136(17)$ & $-0.0279(17)$ & $0.0330(15)$ \\
C1A & $0.0312(10)$ & $0.0281(9)$ & $0.0380(10)$ & $0.0034(7)$ & $-0.0103(8)$ & $0.0037(8)$ \\
O12A & $0.0312(7)$ & $0.0457(8)$ & $0.0549(9)$ & $0.0008(6)$ & $-0.0157(6)$ & $0.0150(7)$ \\
O9A & $0.0504(9)$ & $0.0500(9)$ & $0.0365(8)$ & $0.0156(7)$ & $-0.0163(7)$ & $0.0022(6)$ \\
N8A & $0.0395(9)$ & $0.0367(9)$ & $0.0354(9)$ & $0.0147(8)$ & $-0.0066(7)$ & $0.0016(7)$ \\
C9A & $0.0305(9)$ & $0.0323(10)$ & $0.0344(10)$ & $0.0026(8)$ & $-0.0086(8)$ & $0.0022(8)$ \\
O13A & $0.0475(9)$ & $0.0455(9)$ & $0.0692(11)$ & $0.0064(7)$ & $-0.0221(8)$ & $0.0192(8)$ \\
O7A & $0.0623(10)$ & $0.0355(8)$ & $0.0500(9)$ & $0.0118(7)$ & $-0.0041(8)$ & $-0.0015(7)$ \\
C11A & $0.0325(10)$ & $0.0314(10)$ & $0.0390(11)$ & $0.0029(8)$ & $-0.0126(8)$ & $0.0021(8)$ \\
C7A & $0.0365(10)$ & $0.0396(11)$ & $0.0332(10)$ & $0.0015(8)$ & $-0.0074(8)$ & $-0.0003(8)$ \\
C10A & $0.0294(9)$ & $0.0294(9)$ & $0.0322(10)$ & $0.0035(7)$ & $-0.0095(8)$ & $0.0001(7)$ \\
N14A & $0.0348(9)$ & $0.0306(8)$ & $0.0509(10)$ & $-0.0021(7)$ & $-0.0107(8)$ & $0.0028(7)$ \\
C1A' & $0.0311(10)$ & $0.0357(10)$ & $0.0417(11)$ & $-0.0013(8)$ & $-0.0089(8)$ & $0.0011(8)$
\end{tabular}




\begin{tabular}{|c|c|c|c|c|c|c|}
\hline $\mathrm{C} 2 \mathrm{~A}$ & $0.0347(10)$ & $0.0389(11)$ & $0.0337(10)$ & $-0.0007(8)$ & $-0.0106(8)$ & $0.0039(8)$ \\
\hline $\mathrm{C} 16 \mathrm{~A}$ & $0.0434(11)$ & $0.0382(11)$ & 0.0385 (11) & $0.0031(9)$ & $-0.0106(9)$ & $-0.0069(9)$ \\
\hline $\mathrm{C} 13 \mathrm{~A}$ & $0.0367(10)$ & $0.0343(10)$ & $0.0442(11)$ & $0.0033(8)$ & $-0.0141(9)$ & $0.0051(9)$ \\
\hline $\mathrm{C} 2 \mathrm{~A}^{\prime}$ & $0.0384(10)$ & $0.0316(10)$ & $0.0383(11)$ & $-0.0006(8)$ & $-0.0090(9)$ & $-0.0039(8)$ \\
\hline $\mathrm{C} 3 \mathrm{~A}^{\prime}$ & $0.0532(13)$ & $0.0374(11)$ & $0.0450(12)$ & $-0.0072(10)$ & $-0.0099(10)$ & $0.0053(9)$ \\
\hline $\mathrm{C} 3 \mathrm{~A}$ & $0.0544(13)$ & $0.0573(14)$ & $0.0417(12)$ & $0.0022(11)$ & $-0.0171(11)$ & $0.0096(10)$ \\
\hline $\mathrm{C} 15 \mathrm{~A}$ & $0.0451(12)$ & $0.0357(11)$ & $0.0536(13)$ & $0.0020(9)$ & $-0.0117(10)$ & $-0.0092(9)$ \\
\hline C6A & $0.0553(14)$ & $0.0508(13)$ & $0.0428(13)$ & $0.0082(11)$ & $-0.0045(10)$ & $-0.0073(10)$ \\
\hline O9A' & $0.1098(16)$ & $0.0449(10)$ & $0.0646(12)$ & $0.0027(10)$ & $0.0074(11)$ & $0.0184(9)$ \\
\hline C6 $A^{\prime}$ & $0.0436(12)$ & $0.0444(13)$ & $0.0654(15)$ & $0.0104(10)$ & $0.0025(11)$ & $0.0081(11)$ \\
\hline $\mathrm{C} 4 \mathrm{~A}^{\prime}$ & $0.0549(14)$ & $0.0604(15)$ & $0.0526(14)$ & $-0.0031(12)$ & $0.0070(12)$ & $0.0084(12)$ \\
\hline $\mathrm{C} 4 \mathrm{~A}$ & $0.0676(16)$ & $0.0842(19)$ & $0.0338(12)$ & $-0.0039(14)$ & $-0.0156(11)$ & $0.0072(12)$ \\
\hline C17A & $0.0480(13)$ & $0.0396(12)$ & $0.0730(16)$ & $-0.0069(10)$ & $-0.0091(12)$ & $0.0135(11)$ \\
\hline $\mathrm{C} 5 \mathrm{~A}$ & $0.0637(16)$ & $0.0794(18)$ & $0.0354(12)$ & $-0.0022(14)$ & $-0.0040(11)$ & $-0.0119(12)$ \\
\hline $\mathrm{C} 8 \mathrm{~A}^{\prime}$ & $0.0543(14)$ & $0.0403(13)$ & $0.090(2)$ & $0.0068(11)$ & $-0.0166(14)$ & $0.0033(12)$ \\
\hline $\mathrm{C} 5 \mathrm{~A}^{\prime}$ & $0.0538(15)$ & $0.0607(16)$ & $0.0698(17)$ & $0.0129(12)$ & $0.0144(13)$ & $0.0020(13)$ \\
\hline N8 & $0.0456(10)$ & $0.0458(10)$ & $0.0310(9)$ & $-0.0206(8)$ & $-0.0067(7)$ & $0.0022(7)$ \\
\hline $\mathrm{O} 12$ & $0.0506(9)$ & $0.0326(7)$ & $0.0507(9)$ & $-0.0067(6)$ & $0.0046(7)$ & $0.0040(6)$ \\
\hline $\mathrm{C} 1$ & $0.0315(10)$ & $0.0352(10)$ & $0.0326(10)$ & $-0.0048(8)$ & $-0.0066(8)$ & $0.0031(8)$ \\
\hline $\mathrm{C} 2$ & $0.0374(10)$ & $0.0339(10)$ & $0.0307(10)$ & $-0.0024(8)$ & $-0.0046(8)$ & $0.0006(8)$ \\
\hline $\mathrm{C} 7$ & $0.0418(11)$ & $0.0346(10)$ & $0.0305(10)$ & $-0.0045(8)$ & $-0.0071(8)$ & $0.0015(8)$ \\
\hline O9 & $0.0753(11)$ & $0.0630(10)$ & $0.0348(8)$ & $-0.0380(9)$ & $-0.0126(8)$ & $0.0138(7)$ \\
\hline $\mathrm{O} 13$ & $0.0449(9)$ & $0.0522(9)$ & $0.0619(10)$ & $-0.0192(7)$ & $-0.0023(8)$ & $0.0087(8)$ \\
\hline $\mathrm{C} 10$ & $0.0333(10)$ & $0.0345(10)$ & $0.0293(9)$ & $-0.0060(8)$ & $-0.0088(8)$ & $0.0019(8)$ \\
\hline $\mathrm{C} 11$ & $0.0358(10)$ & $0.0372(11)$ & $0.0365(11)$ & $-0.0057(8)$ & $-0.0049(8)$ & $0.0047(8)$ \\
\hline N14 & $0.0338(9)$ & $0.0466(10)$ & $0.0458(10)$ & $0.0019(7)$ & $-0.0091(8)$ & $0.0045(8)$ \\
\hline C9 & $0.0406(11)$ & $0.0379(11)$ & $0.0318(10)$ & $-0.0099(9)$ & $-0.0070(9)$ & $0.0035(8)$ \\
\hline $\mathrm{C} 13$ & $0.0380(11)$ & $0.0403(11)$ & $0.0372(11)$ & $-0.0088(9)$ & $-0.0081(9)$ & $0.0046(9)$ \\
\hline $\mathrm{C} 1^{\prime}$ & $0.0447(12)$ & $0.0347(11)$ & $0.0440(12)$ & $-0.0009(9)$ & $-0.0041(9)$ & $0.0012(9)$ \\
\hline $\mathrm{C} 3$ & $0.0424(11)$ & $0.0434(12)$ & $0.0374(11)$ & $-0.0054(9)$ & $-0.0021(9)$ & $0.0021(9)$ \\
\hline $\mathrm{C} 4$ & $0.0589(14)$ & $0.0477(12)$ & $0.0298(11)$ & $-0.0007(10)$ & $-0.0028(10)$ & $0.0024(9)$ \\
\hline $\mathrm{C} 2^{\prime}$ & $0.0395(11)$ & $0.0517(12)$ & $0.0386(11)$ & $0.0032(9)$ & $-0.0106(9)$ & $-0.0002(9)$ \\
\hline $\mathrm{C} 16$ & $0.0473(12)$ & $0.0494(12)$ & $0.0385(11)$ & $-0.0036(10)$ & $-0.0184(10)$ & $0.0034(9)$ \\
\hline C6 & $0.0535(13)$ & $0.0474(12)$ & $0.0387(12)$ & $-0.0120(10)$ & $-0.0137(10)$ & $-0.0025(9)$ \\
\hline $\mathrm{C} 5$ & $0.0679(15)$ & $0.0490(13)$ & $0.0326(11)$ & $-0.0033(11)$ & $-0.0167(10)$ & $-0.0033(9)$ \\
\hline O7' & $0.0427(9)$ & $0.153(2)$ & $0.0628(12)$ & $-0.0300(11)$ & $-0.0035(8)$ & $-0.0395(12)$ \\
\hline $\mathrm{C} 15$ & $0.0451(12)$ & $0.0608(14)$ & $0.0531(14)$ & $-0.0037(11)$ & $-0.0234(11)$ & $0.0089(11)$ \\
\hline $\mathrm{C} 3^{\prime}$ & $0.0448(12)$ & $0.0591(14)$ & $0.0462(13)$ & $0.0061(11)$ & $-0.0026(10)$ & $0.0012(11)$ \\
\hline $\mathrm{C} 6^{\prime}$ & $0.0755(19)$ & $0.0697(18)$ & $0.081(2)$ & $-0.0368(15)$ & $0.0209(15)$ & $-0.0341(15)$ \\
\hline O9' & $0.0486(11)$ & $0.178(2)$ & $0.0603(12)$ & $-0.0212(13)$ & $0.0080(9)$ & $-0.0357(14)$ \\
\hline $\mathrm{C} 17$ & $0.0378(12)$ & $0.0619(15)$ & $0.0693(16)$ & $0.0054(11)$ & $-0.0058(11)$ & $0.0029(12)$ \\
\hline $\mathrm{C} 5^{\prime}$ & $0.113(3)$ & $0.090(2)$ & $0.098(2)$ & $-0.056(2)$ & $0.034(2)$ & $-0.056(2)$ \\
\hline $\mathrm{C} 4^{\prime}$ & $0.087(2)$ & $0.0551(16)$ & $0.0642(17)$ & $-0.0073(14)$ & $0.0179(15)$ & $-0.0229(13)$ \\
\hline $\mathrm{C} 8^{\prime}$ & $0.0470(14)$ & $0.0657(17)$ & $0.104(2)$ & $-0.0104(12)$ & $-0.0114(14)$ & $-0.0082(16)$ \\
\hline $\mathrm{C} 10^{\prime}$ & $0.0645(18)$ & $0.103(2)$ & $0.0633(18)$ & $-0.0090(16)$ & $0.0159(14)$ & $-0.0132(16)$ \\
\hline
\end{tabular}


Geometric parameters $\left(\AA,{ }^{\circ}\right)$

\begin{tabular}{|c|c|c|c|}
\hline $\mathrm{C} 10 \mathrm{~B}-\mathrm{O}^{\prime} \mathrm{A}^{\prime}$ & $1.392(3)$ & $\mathrm{N} 8-\mathrm{C} 9$ & $1.344(2)$ \\
\hline $\mathrm{C} 10 \mathrm{~B}-\mathrm{H} 10 \mathrm{~A}$ & 0.9600 & $\mathrm{~N} 8-\mathrm{C} 7$ & $1.403(2)$ \\
\hline $\mathrm{C} 10 \mathrm{~B}-\mathrm{H} 10 \mathrm{~B}$ & 0.9600 & $\mathrm{~N} 8-\mathrm{H} 8$ & $0.89(3)$ \\
\hline $\mathrm{C} 10 \mathrm{~B}-\mathrm{H} 10 \mathrm{C}$ & 0.9600 & $\mathrm{O} 12-\mathrm{C} 13$ & $1.338(2)$ \\
\hline $\mathrm{C} 1 \mathrm{~A}-\mathrm{N} 14 \mathrm{~A}$ & $1.465(2)$ & $\mathrm{O} 12-\mathrm{C} 11$ & $1.457(2)$ \\
\hline $\mathrm{C} 1 \mathrm{~A}-\mathrm{C} 2 \mathrm{~A}$ & $1.506(3)$ & $\mathrm{C} 1-\mathrm{N} 14$ & $1.454(2)$ \\
\hline $\mathrm{C} 1 \mathrm{~A}-\mathrm{C} 10 \mathrm{~A}$ & $1.541(2)$ & $\mathrm{C} 1-\mathrm{C} 2$ & $1.513(3)$ \\
\hline $\mathrm{C} 1 \mathrm{~A}-\mathrm{C} 13 \mathrm{~A}$ & $1.548(3)$ & $\mathrm{C} 1-\mathrm{C} 10$ & $1.535(3)$ \\
\hline $\mathrm{O} 12 \mathrm{~A}-\mathrm{C} 13 \mathrm{~A}$ & $1.341(2)$ & $\mathrm{C} 1-\mathrm{C} 13$ & $1.541(3)$ \\
\hline $\mathrm{O} 12 \mathrm{~A}-\mathrm{C} 11 \mathrm{~A}$ & $1.452(2)$ & $\mathrm{C} 2-\mathrm{C} 3$ & $1.388(3)$ \\
\hline $\mathrm{O} 9 \mathrm{~A}-\mathrm{C} 9 \mathrm{~A}$ & $1.225(2)$ & $\mathrm{C} 2-\mathrm{C} 7$ & $1.389(3)$ \\
\hline $\mathrm{N} 8 \mathrm{~A}-\mathrm{C} 9 \mathrm{~A}$ & $1.343(2)$ & $\mathrm{C} 7-\mathrm{C} 6$ & $1.387(3)$ \\
\hline $\mathrm{N} 8 \mathrm{~A}-\mathrm{C} 7 \mathrm{~A}$ & $1.403(2)$ & $\mathrm{O} 9-\mathrm{C} 9$ & $1.221(2)$ \\
\hline $\mathrm{N} 8 \mathrm{~A}-\mathrm{H} 8 \mathrm{~A}$ & $0.89(2)$ & $\mathrm{O} 13-\mathrm{C} 13$ & $1.197(2)$ \\
\hline $\mathrm{C} 9 \mathrm{~A}-\mathrm{C} 10 \mathrm{~A}$ & $1.512(2)$ & $\mathrm{C} 10-\mathrm{C} 9$ & $1.518(2)$ \\
\hline $\mathrm{O} 13 \mathrm{~A}-\mathrm{C} 13 \mathrm{~A}$ & $1.195(2)$ & $\mathrm{C} 10-\mathrm{C} 16$ & $1.539(3)$ \\
\hline $\mathrm{O} 7 \mathrm{~A}^{\prime}-\mathrm{C} 2 \mathrm{~A}^{\prime}$ & $1.358(2)$ & $\mathrm{C} 10-\mathrm{C} 11$ & $1.546(3)$ \\
\hline $\mathrm{O} 7 \mathrm{~A}^{\prime}-\mathrm{C} 8 \mathrm{~A}^{\prime}$ & $1.422(3)$ & $\mathrm{C} 11-\mathrm{C}^{\prime}$ & $1.499(3)$ \\
\hline $\mathrm{C} 11 \mathrm{~A}-\mathrm{C} 1 \mathrm{~A}^{\prime}$ & $1.499(3)$ & $\mathrm{C} 11-\mathrm{H} 11$ & 0.9800 \\
\hline $\mathrm{C} 11 \mathrm{~A}-\mathrm{C} 10 \mathrm{~A}$ & $1.551(2)$ & $\mathrm{N} 14-\mathrm{C} 17$ & $1.455(3)$ \\
\hline $\mathrm{C} 11 \mathrm{~A}-\mathrm{H} 11 \mathrm{~A}$ & 0.9800 & $\mathrm{~N} 14-\mathrm{C} 15$ & $1.463(3)$ \\
\hline $\mathrm{C} 7 \mathrm{~A}-\mathrm{C} 6 \mathrm{~A}$ & $1.386(3)$ & $\mathrm{C} 1^{\prime}-\mathrm{C} 6^{\prime}$ & $1.381(3)$ \\
\hline $\mathrm{C} 7 \mathrm{~A}-\mathrm{C} 2 \mathrm{~A}$ & $1.390(3)$ & $\mathrm{C} 1^{\prime}-\mathrm{C} 2^{\prime}$ & $1.383(3)$ \\
\hline $\mathrm{C} 10 \mathrm{~A}-\mathrm{C} 16 \mathrm{~A}$ & $1.543(3)$ & $\mathrm{C} 3-\mathrm{C} 4$ & $1.373(3)$ \\
\hline $\mathrm{N} 14 \mathrm{~A}-\mathrm{C} 15 \mathrm{~A}$ & $1.457(3)$ & $\mathrm{C} 3-\mathrm{H} 3$ & 0.9300 \\
\hline $\mathrm{N} 14 \mathrm{~A}-\mathrm{C} 17 \mathrm{~A}$ & $1.459(3)$ & $\mathrm{C} 4-\mathrm{C} 5$ & $1.378(3)$ \\
\hline $\mathrm{C} 1 \mathrm{~A}^{\prime}-\mathrm{C} 6 \mathrm{~A}^{\prime}$ & $1.388(3)$ & $\mathrm{C} 4-\mathrm{H} 4$ & 0.9300 \\
\hline $\mathrm{C} 1 \mathrm{~A}^{\prime}-\mathrm{C} 2 \mathrm{~A}^{\prime}$ & $1.388(3)$ & $\mathrm{C} 2^{\prime}-\mathrm{O} 7^{\prime}$ & $1.343(3)$ \\
\hline $\mathrm{C} 2 \mathrm{~A}-\mathrm{C} 3 \mathrm{~A}$ & $1.390(3)$ & $\mathrm{C} 2^{\prime}-\mathrm{C} 3^{\prime}$ & $1.398(3)$ \\
\hline $\mathrm{C} 16 \mathrm{~A}-\mathrm{C} 15 \mathrm{~A}$ & $1.519(3)$ & $\mathrm{C} 16-\mathrm{C} 15$ & $1.521(3)$ \\
\hline $\mathrm{C} 16 \mathrm{~A}-\mathrm{H} 16 \mathrm{~A}$ & 0.9700 & $\mathrm{C} 16-\mathrm{H} 16 \mathrm{C}$ & 0.9700 \\
\hline $\mathrm{C} 16 \mathrm{~A}-\mathrm{H} 16 \mathrm{~B}$ & 0.9700 & $\mathrm{C} 16-\mathrm{H} 16 \mathrm{D}$ & 0.9700 \\
\hline $\mathrm{C} 2 \mathrm{~A}^{\prime}-\mathrm{C} 3 \mathrm{~A}^{\prime}$ & $1.398(3)$ & $\mathrm{C} 6-\mathrm{C} 5$ & $1.374(3)$ \\
\hline $\mathrm{C} 3 \mathrm{~A}^{\prime}-09 \mathrm{~A}^{\prime}$ & $1.359(3)$ & $\mathrm{C} 6-\mathrm{H} 6$ & 0.9300 \\
\hline $\mathrm{C} 3 \mathrm{~A}^{\prime}-\mathrm{C} 4 \mathrm{~A}^{\prime}$ & $1.374(3)$ & $\mathrm{C} 5-\mathrm{H} 5$ & 0.9300 \\
\hline $\mathrm{C} 3 \mathrm{~A}-\mathrm{C} 4 \mathrm{~A}$ & $1.377(3)$ & $\mathrm{O} 7^{\prime}-\mathrm{C} 8^{\prime}$ & $1.374(3)$ \\
\hline $\mathrm{C} 3 \mathrm{~A}-\mathrm{H} 3 \mathrm{~A}$ & 0.9300 & $\mathrm{C} 15-\mathrm{H} 15 \mathrm{C}$ & 0.9700 \\
\hline $\mathrm{C} 15 \mathrm{~A}-\mathrm{H} 15 \mathrm{~A}$ & 0.9700 & $\mathrm{C} 15-\mathrm{H} 15 \mathrm{D}$ & 0.9700 \\
\hline $\mathrm{C} 15 \mathrm{~A}-\mathrm{H} 15 \mathrm{~B}$ & 0.9700 & $\mathrm{C} 3^{\prime}-\mathrm{O}^{\prime}$ & $1.357(3)$ \\
\hline $\mathrm{C} 6 \mathrm{~A}-\mathrm{C} 5 \mathrm{~A}$ & $1.375(3)$ & $\mathrm{C} 3^{\prime}-\mathrm{C} 4^{\prime}$ & $1.366(4)$ \\
\hline $\mathrm{C} 6 \mathrm{~A}-\mathrm{H} 6 \mathrm{~A}$ & 0.9300 & $\mathrm{C} 6^{\prime}-\mathrm{C}^{\prime}$ & $1.369(4)$ \\
\hline $\mathrm{C} 6 \mathrm{~A}^{\prime}-\mathrm{C} 5 \mathrm{~A}^{\prime}$ & $1.372(3)$ & $\mathrm{C} 6^{\prime}-\mathrm{H} 6^{\prime}$ & 0.9300 \\
\hline $\mathrm{C}^{6} \mathrm{~A}^{\prime}-\mathrm{H}_{6} \mathrm{~A}^{\prime}$ & 0.9300 & $\mathrm{O} 9^{\prime}-\mathrm{C} 10^{\prime}$ & $1.395(3)$ \\
\hline $\mathrm{C} 4 \mathrm{~A}^{\prime}-\mathrm{C} 5 \mathrm{~A}^{\prime}$ & $1.375(4)$ & C17-H17D & 0.9600 \\
\hline $\mathrm{C} 4 \mathrm{~A}^{\prime}-\mathrm{H} 4 \mathrm{~A}^{\prime}$ & 0.9300 & C17-H17E & 0.9600 \\
\hline $\mathrm{C} 4 \mathrm{~A}-\mathrm{C} 5 \mathrm{~A}$ & $1.370(4)$ & $\mathrm{C} 17-\mathrm{H} 17 \mathrm{~F}$ & 0.9600 \\
\hline
\end{tabular}




\begin{tabular}{|c|c|c|c|}
\hline $\mathrm{C} 4 \mathrm{~A}-\mathrm{H} 4 \mathrm{~A}$ & 0.9300 & $\mathrm{C} 5^{\prime}-\mathrm{C} 4^{\prime}$ & $1.366(4)$ \\
\hline $\mathrm{C} 17 \mathrm{~A}-\mathrm{H} 17 \mathrm{~A}$ & 0.9600 & $\mathrm{C} 5^{\prime}-\mathrm{H} 5^{\prime}$ & 0.9300 \\
\hline $\mathrm{C} 17 \mathrm{~A}-\mathrm{H} 17 \mathrm{~B}$ & 0.9600 & $\mathrm{C} 4^{\prime}-\mathrm{H} 4^{\prime}$ & 0.9300 \\
\hline $\mathrm{C} 17 \mathrm{~A}-\mathrm{H} 17 \mathrm{C}$ & 0.9600 & $\mathrm{C} 8^{\prime}-\mathrm{H} 8^{\prime} 1$ & 0.9600 \\
\hline $\mathrm{C} 5 \mathrm{~A}-\mathrm{H} 5 \mathrm{~A}$ & 0.9300 & $\mathrm{C} 8^{\prime}-\mathrm{H} 8^{\prime} 2$ & 0.9600 \\
\hline $\mathrm{C}^{8} \mathrm{~A}^{\prime}-\mathrm{H} 8 \mathrm{~A} 1$ & 0.9600 & $\mathrm{C} 8^{\prime}-\mathrm{H} 8^{\prime} 3$ & 0.9600 \\
\hline $\mathrm{C}^{8} \mathrm{~A}^{\prime}-\mathrm{H} 8 \mathrm{~A} 2$ & 0.9600 & $\mathrm{C} 10^{\prime}-\mathrm{H} 10 \mathrm{D}$ & 0.9600 \\
\hline $\mathrm{C} 8 \mathrm{~A}^{\prime}-\mathrm{H} 8 \mathrm{~A} 3$ & 0.9600 & $\mathrm{C} 10^{\prime}-\mathrm{H} 10 \mathrm{E}$ & 0.9600 \\
\hline $\mathrm{C} 5 \mathrm{~A}^{\prime}-\mathrm{H} 5 \mathrm{~A}^{\prime}$ & 0.9300 & $\mathrm{C} 10^{\prime}-\mathrm{H} 10 \mathrm{~F}$ & 0.9600 \\
\hline $\mathrm{O}^{2} \mathrm{~A}^{\prime}-\mathrm{C} 10 \mathrm{~B}-\mathrm{H} 10 \mathrm{~A}$ & 109.5 & $\mathrm{C} 9-\mathrm{N} 8-\mathrm{C} 7$ & $125.51(16)$ \\
\hline O9A $\mathrm{A}^{\prime}-\mathrm{C} 10 \mathrm{~B}-\mathrm{H} 10 \mathrm{~B}$ & 109.5 & $\mathrm{C} 9-\mathrm{N} 8-\mathrm{H} 8$ & $117.3(16)$ \\
\hline $\mathrm{H} 10 \mathrm{~A}-\mathrm{C} 10 \mathrm{~B}-\mathrm{H} 10 \mathrm{~B}$ & 109.5 & $\mathrm{C} 7-\mathrm{N} 8-\mathrm{H} 8$ & $116.9(16)$ \\
\hline $\mathrm{O}^{2} \mathrm{~A}^{\prime}-\mathrm{C} 10 \mathrm{~B}-\mathrm{H} 10 \mathrm{C}$ & 109.5 & $\mathrm{C} 13-\mathrm{O} 12-\mathrm{C} 11$ & $111.27(15)$ \\
\hline $\mathrm{H} 10 \mathrm{~A}-\mathrm{C} 10 \mathrm{~B}-\mathrm{H} 10 \mathrm{C}$ & 109.5 & $\mathrm{~N} 14-\mathrm{C} 1-\mathrm{C} 2$ & $114.61(16)$ \\
\hline $\mathrm{H} 10 \mathrm{~B}-\mathrm{C} 10 \mathrm{~B}-\mathrm{H} 10 \mathrm{C}$ & 109.5 & $\mathrm{~N} 14-\mathrm{C} 1-\mathrm{C} 10$ & $103.07(15)$ \\
\hline $\mathrm{N} 14 \mathrm{~A}-\mathrm{C} 1 \mathrm{~A}-\mathrm{C} 2 \mathrm{~A}$ & $113.64(15)$ & $\mathrm{C} 2-\mathrm{C} 1-\mathrm{C} 10$ & $113.98(15)$ \\
\hline $\mathrm{N} 14 \mathrm{~A}-\mathrm{C} 1 \mathrm{~A}-\mathrm{C} 10 \mathrm{~A}$ & $102.66(14)$ & $\mathrm{N} 14-\mathrm{C} 1-\mathrm{C} 13$ & $115.71(15)$ \\
\hline $\mathrm{C} 2 \mathrm{~A}-\mathrm{C} 1 \mathrm{~A}-\mathrm{C} 10 \mathrm{~A}$ & $114.65(15)$ & $\mathrm{C} 2-\mathrm{C} 1-\mathrm{C} 13$ & $108.15(15)$ \\
\hline $\mathrm{N} 14 \mathrm{~A}-\mathrm{C} 1 \mathrm{~A}-\mathrm{C} 13 \mathrm{~A}$ & $115.07(15)$ & $\mathrm{C} 10-\mathrm{C} 1-\mathrm{C} 13$ & $100.52(15)$ \\
\hline $\mathrm{C} 2 \mathrm{~A}-\mathrm{C} 1 \mathrm{~A}-\mathrm{C} 13 \mathrm{~A}$ & $109.39(15)$ & $\mathrm{C} 3-\mathrm{C} 2-\mathrm{C} 7$ & $118.31(18)$ \\
\hline $\mathrm{C} 10 \mathrm{~A}-\mathrm{C} 1 \mathrm{~A}-\mathrm{C} 13 \mathrm{~A}$ & $100.71(14)$ & $\mathrm{C} 3-\mathrm{C} 2-\mathrm{C} 1$ & $122.57(17)$ \\
\hline $\mathrm{C} 13 \mathrm{~A}-\mathrm{O} 12 \mathrm{~A}-\mathrm{C} 11 \mathrm{~A}$ & $110.64(14)$ & $\mathrm{C} 7-\mathrm{C} 2-\mathrm{C} 1$ & $119.12(16)$ \\
\hline $\mathrm{C} 9 \mathrm{~A}-\mathrm{N} 8 \mathrm{~A}-\mathrm{C} 7 \mathrm{~A}$ & $126.00(17)$ & $\mathrm{C} 6-\mathrm{C} 7-\mathrm{C} 2$ & $120.67(18)$ \\
\hline $\mathrm{C} 9 \mathrm{~A}-\mathrm{N} 8 \mathrm{~A}-\mathrm{H} 8 \mathrm{~A}$ & $117.9(15)$ & $\mathrm{C} 6-\mathrm{C} 7-\mathrm{N} 8$ & $118.95(17)$ \\
\hline $\mathrm{C} 7 \mathrm{~A}-\mathrm{N} 8 \mathrm{~A}-\mathrm{H} 8 \mathrm{~A}$ & $116.0(15)$ & $\mathrm{C} 2-\mathrm{C} 7-\mathrm{N} 8$ & $120.36(17)$ \\
\hline $\mathrm{O} 9 \mathrm{~A}-\mathrm{C} 9 \mathrm{~A}-\mathrm{N} 8 \mathrm{~A}$ & $121.57(17)$ & $\mathrm{C} 9-\mathrm{C} 10-\mathrm{C} 1$ & $113.04(15)$ \\
\hline $\mathrm{O} 9 \mathrm{~A}-\mathrm{C} 9 \mathrm{~A}-\mathrm{C} 10 \mathrm{~A}$ & $121.93(17)$ & $\mathrm{C} 9-\mathrm{C} 10-\mathrm{C} 16$ & $111.75(15)$ \\
\hline $\mathrm{N} 8 \mathrm{~A}-\mathrm{C} 9 \mathrm{~A}-\mathrm{C} 10 \mathrm{~A}$ & $116.47(16)$ & $\mathrm{C} 1-\mathrm{C} 10-\mathrm{C} 16$ & $102.91(15)$ \\
\hline $\mathrm{C} 2 \mathrm{~A}^{\prime}-\mathrm{O} 7 \mathrm{~A}^{\prime}-\mathrm{C} 8 \mathrm{~A}^{\prime}$ & $121.12(18)$ & $\mathrm{C} 9-\mathrm{C} 10-\mathrm{C} 11$ & $111.71(15)$ \\
\hline $\mathrm{O} 12 \mathrm{~A}-\mathrm{C} 11 \mathrm{~A}-\mathrm{C}^{\prime} \mathrm{A}^{\prime}$ & $109.37(15)$ & $\mathrm{C} 1-\mathrm{C} 10-\mathrm{C} 11$ & $102.04(14)$ \\
\hline $\mathrm{O} 12 \mathrm{~A}-\mathrm{C} 11 \mathrm{~A}-\mathrm{C} 10 \mathrm{~A}$ & $103.00(14)$ & $\mathrm{C} 16-\mathrm{C} 10-\mathrm{C} 11$ & $114.72(16)$ \\
\hline $\mathrm{C} 1 \mathrm{~A}^{\prime}-\mathrm{C} 11 \mathrm{~A}-\mathrm{C} 10 \mathrm{~A}$ & $117.58(15)$ & $\mathrm{O} 12-\mathrm{C} 11-\mathrm{C}^{\prime}$ & $108.71(15)$ \\
\hline $\mathrm{O} 12 \mathrm{~A}-\mathrm{C} 11 \mathrm{~A}-\mathrm{H} 11 \mathrm{~A}$ & 108.8 & $\mathrm{O} 12-\mathrm{C} 11-\mathrm{C} 10$ & $102.87(15)$ \\
\hline $\mathrm{C} 1 \mathrm{~A}^{\prime}-\mathrm{C} 11 \mathrm{~A}-\mathrm{H} 11 \mathrm{~A}$ & 108.8 & $\mathrm{C} 11^{\prime}-\mathrm{C} 11-\mathrm{C} 10$ & $117.93(16)$ \\
\hline $\mathrm{C} 10 \mathrm{~A}-\mathrm{C} 11 \mathrm{~A}-\mathrm{H} 11 \mathrm{~A}$ & 108.8 & $\mathrm{O} 12-\mathrm{C} 11-\mathrm{H} 11$ & 109.0 \\
\hline $\mathrm{C} 6 \mathrm{~A}-\mathrm{C} 7 \mathrm{~A}-\mathrm{C} 2 \mathrm{~A}$ & $120.61(18)$ & $\mathrm{C} 1^{\prime}-\mathrm{C} 11-\mathrm{H} 11$ & 109.0 \\
\hline $\mathrm{C} 6 \mathrm{~A}-\mathrm{C} 7 \mathrm{~A}-\mathrm{N} 8 \mathrm{~A}$ & $118.81(18)$ & $\mathrm{C} 10-\mathrm{C} 11-\mathrm{H} 11$ & 109.0 \\
\hline $\mathrm{C} 2 \mathrm{~A}-\mathrm{C} 7 \mathrm{~A}-\mathrm{N} 8 \mathrm{~A}$ & $120.56(17)$ & $\mathrm{C} 1-\mathrm{N} 14-\mathrm{C} 17$ & $118.80(17)$ \\
\hline $\mathrm{C} 9 \mathrm{~A}-\mathrm{C} 10 \mathrm{~A}-\mathrm{C} 1 \mathrm{~A}$ & $114.39(15)$ & $\mathrm{C} 1-\mathrm{N} 14-\mathrm{C} 15$ & $104.91(16)$ \\
\hline $\mathrm{C} 9 \mathrm{~A}-\mathrm{C} 10 \mathrm{~A}-\mathrm{C} 16 \mathrm{~A}$ & $111.90(15)$ & C17-N14-C15 & $113.96(18)$ \\
\hline $\mathrm{C} 1 \mathrm{~A}-\mathrm{C} 10 \mathrm{~A}-\mathrm{C} 16 \mathrm{~A}$ & $103.25(14)$ & $\mathrm{O} 9-\mathrm{C} 9-\mathrm{N} 8$ & $121.40(17)$ \\
\hline $\mathrm{C} 9 \mathrm{~A}-\mathrm{C} 10 \mathrm{~A}-\mathrm{C} 11 \mathrm{~A}$ & $110.44(14)$ & $\mathrm{O} 9-\mathrm{C} 9-\mathrm{C} 10$ & $122.32(17)$ \\
\hline $\mathrm{C} 1 \mathrm{~A}-\mathrm{C} 10 \mathrm{~A}-\mathrm{C} 11 \mathrm{~A}$ & $101.78(14)$ & $\mathrm{N} 8-\mathrm{C} 9-\mathrm{C} 10$ & $116.26(16)$ \\
\hline $\mathrm{C} 16 \mathrm{~A}-\mathrm{C} 10 \mathrm{~A}-\mathrm{C} 11 \mathrm{~A}$ & $114.60(15)$ & $\mathrm{O} 13-\mathrm{C} 13-\mathrm{O} 12$ & $121.44(19)$ \\
\hline $\mathrm{C} 15 \mathrm{~A}-\mathrm{N} 14 \mathrm{~A}-\mathrm{C} 17 \mathrm{~A}$ & $113.06(17)$ & $\mathrm{O} 13-\mathrm{C} 13-\mathrm{C} 1$ & $128.59(19)$ \\
\hline $\mathrm{C} 15 \mathrm{~A}-\mathrm{N} 14 \mathrm{~A}-\mathrm{C} 1 \mathrm{~A}$ & $105.39(15)$ & $\mathrm{O} 12-\mathrm{C} 13-\mathrm{C} 1$ & $109.97(15)$ \\
\hline $\mathrm{C} 17 \mathrm{~A}-\mathrm{N} 14 \mathrm{~A}-\mathrm{C} 1 \mathrm{~A}$ & $118.68(17)$ & $\mathrm{C} 6^{\prime}-\mathrm{C} 1^{\prime}-\mathrm{C} 2^{\prime}$ & $119.2(2)$ \\
\hline
\end{tabular}




\begin{tabular}{|c|c|}
\hline $\mathrm{C} 6 \mathrm{~A}^{\prime}-\mathrm{C} 1 \mathrm{~A}^{\prime}-\mathrm{C} 2 \mathrm{~A}^{\prime}$ & $119.18(19)$ \\
\hline $\mathrm{C}^{6} \mathrm{~A}^{\prime}-\mathrm{C} 1 \mathrm{~A}^{\prime}-\mathrm{C} 11 \mathrm{~A}$ & $121.77(18)$ \\
\hline $\mathrm{C} 2 \mathrm{~A}^{\prime}-\mathrm{C} 1 \mathrm{~A}^{\prime}-\mathrm{C} 11 \mathrm{~A}$ & $119.05(17)$ \\
\hline $\mathrm{C} 7 \mathrm{~A}-\mathrm{C} 2 \mathrm{~A}-\mathrm{C} 3 \mathrm{~A}$ & $118.03(19)$ \\
\hline $\mathrm{C} 7 \mathrm{~A}-\mathrm{C} 2 \mathrm{~A}-\mathrm{C} 1 \mathrm{~A}$ & $119.21(16)$ \\
\hline $\mathrm{C} 3 \mathrm{~A}-\mathrm{C} 2 \mathrm{~A}-\mathrm{C} 1 \mathrm{~A}$ & $122.74(18)$ \\
\hline $\mathrm{C} 15 \mathrm{~A}-\mathrm{C} 16 \mathrm{~A}-\mathrm{C} 10 \mathrm{~A}$ & $105.86(16)$ \\
\hline $\mathrm{C} 15 \mathrm{~A}-\mathrm{C} 16 \mathrm{~A}-\mathrm{H} 16 \mathrm{~A}$ & 110.6 \\
\hline $\mathrm{C} 10 \mathrm{~A}-\mathrm{C} 16 \mathrm{~A}-\mathrm{H} 16 \mathrm{~A}$ & 110.6 \\
\hline $\mathrm{C} 15 \mathrm{~A}-\mathrm{C} 16 \mathrm{~A}-\mathrm{H} 16 \mathrm{~B}$ & 110.6 \\
\hline $\mathrm{C} 10 \mathrm{~A}-\mathrm{C} 16 \mathrm{~A}-\mathrm{H} 16 \mathrm{~B}$ & 110.6 \\
\hline $\mathrm{H} 16 \mathrm{~A}-\mathrm{C} 16 \mathrm{~A}-\mathrm{H} 16 \mathrm{~B}$ & 108.7 \\
\hline $\mathrm{O} 13 \mathrm{~A}-\mathrm{C} 13 \mathrm{~A}-\mathrm{O} 12 \mathrm{~A}$ & $121.52(18)$ \\
\hline $\mathrm{O} 13 \mathrm{~A}-\mathrm{C} 13 \mathrm{~A}-\mathrm{C} 1 \mathrm{~A}$ & $128.10(18)$ \\
\hline $\mathrm{O} 12 \mathrm{~A}-\mathrm{C} 13 \mathrm{~A}-\mathrm{C} 1 \mathrm{~A}$ & $110.37(15)$ \\
\hline $\mathrm{O}^{2} \mathrm{~A}^{\prime}-\mathrm{C} 2 \mathrm{~A}^{\prime}-\mathrm{C}^{\prime} \mathrm{A}^{\prime}$ & $115.13(17)$ \\
\hline $\mathrm{O} 7 \mathrm{~A}^{\prime}-\mathrm{C} 2 \mathrm{~A}^{\prime}-\mathrm{C} 3 \mathrm{~A}^{\prime}$ & $124.65(18)$ \\
\hline $\mathrm{C} 1 \mathrm{~A}^{\prime}-\mathrm{C} 2 \mathrm{~A}^{\prime}-\mathrm{C} 3 \mathrm{~A}^{\prime}$ & $119.92(19)$ \\
\hline $\mathrm{O} 9 \mathrm{~A}^{\prime}-\mathrm{C} 3 \mathrm{~A}^{\prime}-\mathrm{C} 4 \mathrm{~A}^{\prime}$ & $122.8(2)$ \\
\hline $\mathrm{O} 9 \mathrm{~A}^{\prime}-\mathrm{C} 3 \mathrm{~A}^{\prime}-\mathrm{C} 2 \mathrm{~A}^{\prime}$ & $117.4(2)$ \\
\hline $\mathrm{C} 4 \mathrm{~A}^{\prime}-\mathrm{C} 3 \mathrm{~A}^{\prime}-\mathrm{C} 2 \mathrm{~A}^{\prime}$ & $119.8(2)$ \\
\hline $\mathrm{C} 4 \mathrm{~A}-\mathrm{C} 3 \mathrm{~A}-\mathrm{C} 2 \mathrm{~A}$ & $121.3(2)$ \\
\hline $\mathrm{C} 4 \mathrm{~A}-\mathrm{C} 3 \mathrm{~A}-\mathrm{H} 3 \mathrm{~A}$ & 119.4 \\
\hline $\mathrm{C} 2 \mathrm{~A}-\mathrm{C} 3 \mathrm{~A}-\mathrm{H} 3 \mathrm{~A}$ & 119.4 \\
\hline $\mathrm{N} 14 \mathrm{~A}-\mathrm{C} 15 \mathrm{~A}-\mathrm{C} 16 \mathrm{~A}$ & $104.53(16)$ \\
\hline $\mathrm{N} 14 \mathrm{~A}-\mathrm{C} 15 \mathrm{~A}-\mathrm{H} 15 \mathrm{~A}$ & 110.8 \\
\hline $\mathrm{C} 16 \mathrm{~A}-\mathrm{C} 15 \mathrm{~A}-\mathrm{H} 15 \mathrm{~A}$ & 110.8 \\
\hline $\mathrm{N} 14 \mathrm{~A}-\mathrm{C} 15 \mathrm{~A}-\mathrm{H} 15 \mathrm{~B}$ & 110.8 \\
\hline $\mathrm{C} 16 \mathrm{~A}-\mathrm{C} 15 \mathrm{~A}-\mathrm{H} 15 \mathrm{~B}$ & 110.8 \\
\hline $\mathrm{H} 15 \mathrm{~A}-\mathrm{C} 15 \mathrm{~A}-\mathrm{H} 15 \mathrm{~B}$ & 108.9 \\
\hline $\mathrm{C} 5 \mathrm{~A}-\mathrm{C} 6 \mathrm{~A}-\mathrm{C} 7 \mathrm{~A}$ & $119.9(2)$ \\
\hline $\mathrm{C} 5 \mathrm{~A}-\mathrm{C} 6 \mathrm{~A}-\mathrm{H} 6 \mathrm{~A}$ & 120.0 \\
\hline $\mathrm{C} 7 \mathrm{~A}-\mathrm{C} 6 \mathrm{~A}-\mathrm{H} 6 \mathrm{~A}$ & 120.0 \\
\hline $\mathrm{C} 3 \mathrm{~A}^{\prime}-\mathrm{O} 9 \mathrm{~A}^{\prime}-\mathrm{C} 10 \mathrm{~B}$ & $119.3(2)$ \\
\hline $\mathrm{C} 5 \mathrm{~A}^{\prime}-\mathrm{C} 6 \mathrm{~A}^{\prime}-\mathrm{C} 1 \mathrm{~A}^{\prime}$ & $120.4(2)$ \\
\hline $\mathrm{C} 5 \mathrm{~A}^{\prime}-\mathrm{C} 6 \mathrm{~A}^{\prime}-\mathrm{H} 6 \mathrm{~A}^{\prime}$ & 119.8 \\
\hline $\mathrm{C} 1 \mathrm{~A}^{\prime}-\mathrm{C} 6 \mathrm{~A}^{\prime}-\mathrm{H} 6 \mathrm{~A}^{\prime}$ & 119.8 \\
\hline $\mathrm{C} 3 \mathrm{~A}^{\prime}-\mathrm{C} 4 \mathrm{~A}^{\prime}-\mathrm{C} 5 \mathrm{~A}^{\prime}$ & $120.2(2)$ \\
\hline $\mathrm{C} 3 \mathrm{~A}^{\prime}-\mathrm{C} 4 \mathrm{~A}^{\prime}-\mathrm{H} 4 \mathrm{~A}^{\prime}$ & 119.9 \\
\hline $\mathrm{C} 5 \mathrm{~A}^{\prime}-\mathrm{C} 4 \mathrm{~A}^{\prime}-\mathrm{H} 4 \mathrm{~A}^{\prime}$ & 119.9 \\
\hline $\mathrm{C} 5 \mathrm{~A}-\mathrm{C} 4 \mathrm{~A}-\mathrm{C} 3 \mathrm{~A}$ & $119.8(2)$ \\
\hline $\mathrm{C} 5 \mathrm{~A}-\mathrm{C} 4 \mathrm{~A}-\mathrm{H} 4 \mathrm{~A}$ & 120.1 \\
\hline $\mathrm{C} 3 \mathrm{~A}-\mathrm{C} 4 \mathrm{~A}-\mathrm{H} 4 \mathrm{~A}$ & 120.1 \\
\hline $\mathrm{N} 14 \mathrm{~A}-\mathrm{C} 17 \mathrm{~A}-\mathrm{H} 17 \mathrm{~A}$ & 109.5 \\
\hline $\mathrm{N} 14 \mathrm{~A}-\mathrm{C} 17 \mathrm{~A}-\mathrm{H} 17 \mathrm{~B}$ & 109.5 \\
\hline $\mathrm{H} 17 \mathrm{~A}-\mathrm{C} 17 \mathrm{~A}-\mathrm{H} 17 \mathrm{~B}$ & 109.5 \\
\hline N14A-C17A-H17C & 109.5 \\
\hline $\mathrm{H} 17 \mathrm{~A}-\mathrm{C} 17 \mathrm{~A}-\mathrm{H} 17 \mathrm{C}$ & 109.5 \\
\hline
\end{tabular}

\begin{tabular}{|c|c|}
\hline $\mathrm{C} 66^{\prime}-\mathrm{C} 1{ }^{\prime}-\mathrm{C} 11$ & $121.6(2)$ \\
\hline $\mathrm{C} 22^{\prime}-\mathrm{C} 1{ }^{\prime}-\mathrm{C} 11$ & $119.20(18)$ \\
\hline $\mathrm{C} 4-\mathrm{C} 3-\mathrm{C} 2$ & $121.04(19)$ \\
\hline $\mathrm{C} 4-\mathrm{C} 3-\mathrm{H} 3$ & 119.5 \\
\hline $\mathrm{C} 2-\mathrm{C} 3-\mathrm{H} 3$ & 119.5 \\
\hline $\mathrm{C} 3-\mathrm{C} 4-\mathrm{C} 5$ & $120.03(19)$ \\
\hline $\mathrm{C} 3-\mathrm{C} 4-\mathrm{H} 4$ & 120.0 \\
\hline $\mathrm{C} 5-\mathrm{C} 4-\mathrm{H} 4$ & 120.0 \\
\hline $\mathrm{O} 7^{\prime}-\mathrm{C} 2^{\prime}-\mathrm{C} 1^{\prime}$ & $113.90(19)$ \\
\hline $\mathrm{O} 7^{\prime}-\mathrm{C} 2^{\prime}-\mathrm{C} 3^{\prime}$ & $125.9(2)$ \\
\hline $\mathrm{C} 1^{\prime}-\mathrm{C} 2^{\prime}-\mathrm{C} 3^{\prime}$ & $120.1(2)$ \\
\hline $\mathrm{C} 15-\mathrm{C} 16-\mathrm{C} 10$ & $105.64(16)$ \\
\hline $\mathrm{C} 15-\mathrm{C} 16-\mathrm{H} 16 \mathrm{C}$ & 110.6 \\
\hline $\mathrm{C} 10-\mathrm{C} 16-\mathrm{H} 16 \mathrm{C}$ & 110.6 \\
\hline $\mathrm{C} 15-\mathrm{C} 16-\mathrm{H} 16 \mathrm{D}$ & 110.6 \\
\hline $\mathrm{C} 10-\mathrm{C} 16-\mathrm{H} 16 \mathrm{D}$ & 110.6 \\
\hline $\mathrm{H} 16 \mathrm{C}-\mathrm{C} 16-\mathrm{H} 16 \mathrm{D}$ & 108.7 \\
\hline $\mathrm{C} 5-\mathrm{C} 6-\mathrm{C} 7$ & $119.8(2)$ \\
\hline $\mathrm{C} 5-\mathrm{C} 6-\mathrm{H} 6$ & 120.1 \\
\hline $\mathrm{C} 7-\mathrm{C} 6-\mathrm{H} 6$ & 120.1 \\
\hline $\mathrm{C} 6-\mathrm{C} 5-\mathrm{C} 4$ & $120.1(2)$ \\
\hline $\mathrm{C} 6-\mathrm{C} 5-\mathrm{H} 5$ & 120.0 \\
\hline $\mathrm{C} 4-\mathrm{C} 5-\mathrm{H} 5$ & 120.0 \\
\hline $\mathrm{C} 22^{\prime}-\mathrm{O} 7^{\prime}-\mathrm{C} 8^{\prime}$ & $126.5(2)$ \\
\hline $\mathrm{N} 14-\mathrm{C} 15-\mathrm{C} 16$ & $104.96(16)$ \\
\hline $\mathrm{N} 14-\mathrm{C} 15-\mathrm{H} 15 \mathrm{C}$ & 110.8 \\
\hline $\mathrm{C} 16-\mathrm{C} 15-\mathrm{H} 15 \mathrm{C}$ & 110.8 \\
\hline N14-C15-H15D & 110.8 \\
\hline $\mathrm{C} 16-\mathrm{C} 15-\mathrm{H} 15 \mathrm{D}$ & 110.8 \\
\hline $\mathrm{H} 15 \mathrm{C}-\mathrm{C} 15-\mathrm{H} 15 \mathrm{D}$ & 108.8 \\
\hline $\mathrm{O} 9^{\prime}-\mathrm{C} 3^{\prime}-\mathrm{C} 4^{\prime}$ & $122.5(2)$ \\
\hline $\mathrm{O} 9^{\prime}-\mathrm{C} 3^{\prime}-\mathrm{C} 2^{\prime}$ & $118.2(2)$ \\
\hline $\mathrm{C} 4^{\prime}-\mathrm{C} 3^{\prime}-\mathrm{C} 2^{\prime}$ & $119.4(2)$ \\
\hline $\mathrm{C} 5^{\prime}-\mathrm{C} 6^{\prime}-\mathrm{C} 1^{\prime}$ & $120.0(2)$ \\
\hline $\mathrm{C} 5^{\prime}-\mathrm{C} 6^{\prime}-\mathrm{H} 6^{\prime}$ & 120.0 \\
\hline $\mathrm{C} 1^{\prime}-\mathrm{C} 6^{\prime}-\mathrm{H} 6^{\prime}$ & 120.0 \\
\hline $\mathrm{C} 3^{\prime}-\mathrm{O}^{\prime}-\mathrm{C} 10^{\prime}$ & $119.0(2)$ \\
\hline N14-C17-H17D & 109.5 \\
\hline $\mathrm{N} 14-\mathrm{C} 17-\mathrm{H} 17 \mathrm{E}$ & 109.5 \\
\hline $\mathrm{H} 17 \mathrm{D}-\mathrm{C} 17-\mathrm{H} 17 \mathrm{E}$ & 109.5 \\
\hline $\mathrm{N} 14-\mathrm{C} 17-\mathrm{H} 17 \mathrm{~F}$ & 109.5 \\
\hline $\mathrm{H} 17 \mathrm{D}-\mathrm{C} 17-\mathrm{H} 17 \mathrm{~F}$ & 109.5 \\
\hline $\mathrm{H} 17 \mathrm{E}-\mathrm{C} 17-\mathrm{H} 17 \mathrm{~F}$ & 109.5 \\
\hline $\mathrm{C} 4^{\prime}-\mathrm{C} 5^{\prime}-\mathrm{C} 6^{\prime}$ & $120.9(3)$ \\
\hline $\mathrm{C} 4^{\prime}-\mathrm{C} 5^{\prime}-\mathrm{H} 5^{\prime}$ & 119.6 \\
\hline $\mathrm{C} 6^{\prime}-\mathrm{C} 5^{\prime}-\mathrm{H} 5^{\prime}$ & 119.6 \\
\hline $\mathrm{C} 3^{\prime}-\mathrm{C} 4^{\prime}-\mathrm{C} 5^{\prime}$ & $120.3(2)$ \\
\hline $\mathrm{C} 3^{\prime}-\mathrm{C} 4^{\prime}-\mathrm{H} 4^{\prime}$ & 119.8 \\
\hline
\end{tabular}




\begin{tabular}{|c|c|c|c|}
\hline $\mathrm{H} 17 \mathrm{~B}-\mathrm{C} 17 \mathrm{~A}-\mathrm{H} 17 \mathrm{C}$ & 109.5 & $\mathrm{C} 5^{\prime}-\mathrm{C} 4^{\prime}-\mathrm{H} 4^{\prime}$ & 119.8 \\
\hline $\mathrm{C} 4 \mathrm{~A}-\mathrm{C} 5 \mathrm{~A}-\mathrm{C} 6 \mathrm{~A}$ & $120.3(2)$ & $\mathrm{O} 7^{\prime}-\mathrm{C} 8^{\prime}-\mathrm{H} 8^{\prime} 1$ & 109.5 \\
\hline $\mathrm{C} 4 \mathrm{~A}-\mathrm{C} 5 \mathrm{~A}-\mathrm{H} 5 \mathrm{~A}$ & 119.8 & $\mathrm{O} 7^{\prime}-\mathrm{C} 8^{\prime}-\mathrm{H} 8^{\prime} 2$ & 109.5 \\
\hline $\mathrm{C} 6 \mathrm{~A}-\mathrm{C} 5 \mathrm{~A}-\mathrm{H} 5 \mathrm{~A}$ & 119.8 & $\mathrm{H} 8^{\prime} 1-\mathrm{C} 8^{\prime}-\mathrm{H} 8^{\prime} 2$ & 109.5 \\
\hline $\mathrm{O}^{\prime} \mathrm{A}^{\prime}-\mathrm{C} 8 \mathrm{~A}^{\prime}-\mathrm{H} 8 \mathrm{~A} 1$ & 109.5 & $\mathrm{O} 7^{\prime}-\mathrm{C} 8^{\prime}-\mathrm{H} 8^{\prime} 3$ & 109.5 \\
\hline $\mathrm{O}^{2} \mathrm{~A}^{\prime}-\mathrm{C} 8 \mathrm{~A}^{\prime}-\mathrm{H} 8 \mathrm{~A} 2$ & 109.5 & $\mathrm{H} 8^{\prime} 1-\mathrm{C} 8^{\prime}-\mathrm{H}^{\prime} 3$ & 109.5 \\
\hline $\mathrm{H} 8 \mathrm{~A} 1-\mathrm{C} 8 \mathrm{~A}^{\prime}-\mathrm{H} 8 \mathrm{~A} 2$ & 109.5 & $\mathrm{H} 8^{\prime} 2-\mathrm{C}^{\prime}-\mathrm{H}^{\prime} 3$ & 109.5 \\
\hline $\mathrm{O}^{\prime} \mathrm{A}^{\prime}-\mathrm{C} 8 \mathrm{~A}^{\prime}-\mathrm{H} 8 \mathrm{~A} 3$ & 109.5 & $\mathrm{O}^{\prime}-\mathrm{C} 10^{\prime}-\mathrm{H} 10 \mathrm{D}$ & 109.5 \\
\hline $\mathrm{H} 8 \mathrm{~A} 1-\mathrm{C} 8 \mathrm{~A}^{\prime}-\mathrm{H} 8 \mathrm{~A} 3$ & 109.5 & $\mathrm{O}^{\prime}-\mathrm{C} 10^{\prime}-\mathrm{H} 10 \mathrm{E}$ & 109.5 \\
\hline $\mathrm{H} 8 \mathrm{~A} 2-\mathrm{C} 8 \mathrm{~A}^{\prime}-\mathrm{H} 8 \mathrm{~A} 3$ & 109.5 & $\mathrm{H} 10 \mathrm{D}-\mathrm{C} 10^{\prime}-\mathrm{H} 10 \mathrm{E}$ & 109.5 \\
\hline $\mathrm{C} 6 \mathrm{~A}^{\prime}-\mathrm{C} 5 \mathrm{~A}^{\prime}-\mathrm{C} 4 \mathrm{~A}^{\prime}$ & $120.5(2)$ & $\mathrm{O}^{\prime}-\mathrm{C} 10^{\prime}-\mathrm{H} 10 \mathrm{~F}$ & 109.5 \\
\hline $\mathrm{C} 6 \mathrm{~A}^{\prime}-\mathrm{C} 5 \mathrm{~A}^{\prime}-\mathrm{H} 5 \mathrm{~A}^{\prime}$ & 119.7 & $\mathrm{H} 10 \mathrm{D}-\mathrm{C} 10^{\prime}-\mathrm{H} 10 \mathrm{~F}$ & 109.5 \\
\hline $\mathrm{C} 4 \mathrm{~A}^{\prime}-\mathrm{C} 5 \mathrm{~A}^{\prime}-\mathrm{H} 5 \mathrm{~A}^{\prime}$ & 119.7 & $\mathrm{H} 10 \mathrm{E}-\mathrm{C} 10^{\prime}-\mathrm{H} 10 \mathrm{~F}$ & 109.5 \\
\hline $\mathrm{C} 7 \mathrm{~A}-\mathrm{N} 8 \mathrm{~A}-\mathrm{C} 9 \mathrm{~A}-\mathrm{O} 9 \mathrm{~A}$ & $169.07(18)$ & $\mathrm{N} 14-\mathrm{C} 1-\mathrm{C} 2-\mathrm{C} 3$ & $-82.4(2)$ \\
\hline $\mathrm{C} 7 \mathrm{~A}-\mathrm{N} 8 \mathrm{~A}-\mathrm{C} 9 \mathrm{~A}-\mathrm{C} 10 \mathrm{~A}$ & $-12.8(3)$ & $\mathrm{C} 10-\mathrm{C} 1-\mathrm{C} 2-\mathrm{C} 3$ & $159.17(18)$ \\
\hline $\mathrm{C} 13 \mathrm{~A}-\mathrm{O} 12 \mathrm{~A}-\mathrm{C} 11 \mathrm{~A}-\mathrm{C} 1 \mathrm{~A}^{\prime}$ & $-153.09(16)$ & $\mathrm{C} 13-\mathrm{C} 1-\mathrm{C} 2-\mathrm{C} 3$ & $48.3(2)$ \\
\hline $\mathrm{C} 13 \mathrm{~A}-\mathrm{O} 12 \mathrm{~A}-\mathrm{C} 11 \mathrm{~A}-\mathrm{C} 10 \mathrm{~A}$ & $-27.3(2)$ & $\mathrm{N} 14-\mathrm{C} 1-\mathrm{C} 2-\mathrm{C} 7$ & $98.4(2)$ \\
\hline $\mathrm{C} 9 \mathrm{~A}-\mathrm{N} 8 \mathrm{~A}-\mathrm{C} 7 \mathrm{~A}-\mathrm{C} 6 \mathrm{~A}$ & $177.86(19)$ & $\mathrm{C} 10-\mathrm{C} 1-\mathrm{C} 2-\mathrm{C} 7$ & $-20.0(3)$ \\
\hline $\mathrm{C} 9 \mathrm{~A}-\mathrm{N} 8 \mathrm{~A}-\mathrm{C} 7 \mathrm{~A}-\mathrm{C} 2 \mathrm{~A}$ & $-4.0(3)$ & $\mathrm{C} 13-\mathrm{C} 1-\mathrm{C} 2-\mathrm{C} 7$ & $-130.91(18)$ \\
\hline $\mathrm{O} 9 \mathrm{~A}-\mathrm{C} 9 \mathrm{~A}-\mathrm{C} 10 \mathrm{~A}-\mathrm{C} 1 \mathrm{~A}$ & $-150.95(18)$ & $\mathrm{C} 3-\mathrm{C} 2-\mathrm{C} 7-\mathrm{C} 6$ & $0.1(3)$ \\
\hline $\mathrm{N} 8 \mathrm{~A}-\mathrm{C} 9 \mathrm{~A}-\mathrm{C} 10 \mathrm{~A}-\mathrm{C} 1 \mathrm{~A}$ & $30.9(2)$ & $\mathrm{C} 1-\mathrm{C} 2-\mathrm{C} 7-\mathrm{C} 6$ & $179.38(19)$ \\
\hline $\mathrm{O} 9 \mathrm{~A}-\mathrm{C} 9 \mathrm{~A}-\mathrm{C} 10 \mathrm{~A}-\mathrm{C} 16 \mathrm{~A}$ & $-34.0(2)$ & $\mathrm{C} 3-\mathrm{C} 2-\mathrm{C} 7-\mathrm{N} 8$ & $178.38(18)$ \\
\hline $\mathrm{N} 8 \mathrm{~A}-\mathrm{C} 9 \mathrm{~A}-\mathrm{C} 10 \mathrm{~A}-\mathrm{C} 16 \mathrm{~A}$ & $147.90(17)$ & $\mathrm{C} 1-\mathrm{C} 2-\mathrm{C} 7-\mathrm{N} 8$ & $-2.4(3)$ \\
\hline $\mathrm{O} 9 \mathrm{~A}-\mathrm{C} 9 \mathrm{~A}-\mathrm{C} 10 \mathrm{~A}-\mathrm{C} 11 \mathrm{~A}$ & $95.0(2)$ & $\mathrm{C} 9-\mathrm{N} 8-\mathrm{C} 7-\mathrm{C} 6$ & $-175.7(2)$ \\
\hline $\mathrm{N} 8 \mathrm{~A}-\mathrm{C} 9 \mathrm{~A}-\mathrm{C} 10 \mathrm{~A}-\mathrm{C} 11 \mathrm{~A}$ & $-83.2(2)$ & $\mathrm{C} 9-\mathrm{N} 8-\mathrm{C} 7-\mathrm{C} 2$ & $6.0(3)$ \\
\hline $\mathrm{N} 14 \mathrm{~A}-\mathrm{C} 1 \mathrm{~A}-\mathrm{C} 10 \mathrm{~A}-\mathrm{C} 9 \mathrm{~A}$ & $90.48(17)$ & $\mathrm{N} 14-\mathrm{C} 1-\mathrm{C} 10-\mathrm{C} 9$ & $-86.85(18)$ \\
\hline $\mathrm{C} 2 \mathrm{~A}-\mathrm{C} 1 \mathrm{~A}-\mathrm{C} 10 \mathrm{~A}-\mathrm{C} 9 \mathrm{~A}$ & $-33.2(2)$ & $\mathrm{C} 2-\mathrm{C} 1-\mathrm{C} 10-\mathrm{C} 9$ & $38.0(2)$ \\
\hline $\mathrm{C} 13 \mathrm{~A}-\mathrm{C} 1 \mathrm{~A}-\mathrm{C} 10 \mathrm{~A}-\mathrm{C} 9 \mathrm{~A}$ & $-150.54(15)$ & $\mathrm{C} 13-\mathrm{C} 1-\mathrm{C} 10-\mathrm{C} 9$ & $153.41(16)$ \\
\hline $\mathrm{N} 14 \mathrm{~A}-\mathrm{C} 1 \mathrm{~A}-\mathrm{C} 10 \mathrm{~A}-\mathrm{C} 16 \mathrm{~A}$ & $-31.36(17)$ & $\mathrm{N} 14-\mathrm{C} 1-\mathrm{C} 10-\mathrm{C} 16$ & $33.86(18)$ \\
\hline $\mathrm{C} 2 \mathrm{~A}-\mathrm{C} 1 \mathrm{~A}-\mathrm{C} 10 \mathrm{~A}-\mathrm{C} 16 \mathrm{~A}$ & $-155.09(15)$ & $\mathrm{C} 2-\mathrm{C} 1-\mathrm{C} 10-\mathrm{C} 16$ & $158.69(16)$ \\
\hline $\mathrm{C} 13 \mathrm{~A}-\mathrm{C} 1 \mathrm{~A}-\mathrm{C} 10 \mathrm{~A}-\mathrm{C} 16 \mathrm{~A}$ & $87.62(16)$ & $\mathrm{C} 13-\mathrm{C} 1-\mathrm{C} 10-\mathrm{C} 16$ & $-85.88(17)$ \\
\hline $\mathrm{N} 14 \mathrm{~A}-\mathrm{C} 1 \mathrm{~A}-\mathrm{C} 10 \mathrm{~A}-\mathrm{C} 11 \mathrm{~A}$ & $-150.43(14)$ & $\mathrm{N} 14-\mathrm{C} 1-\mathrm{C} 10-\mathrm{C} 11$ & $153.04(14)$ \\
\hline $\mathrm{C} 2 \mathrm{~A}-\mathrm{C} 1 \mathrm{~A}-\mathrm{C} 10 \mathrm{~A}-\mathrm{C} 11 \mathrm{~A}$ & $85.84(17)$ & $\mathrm{C} 2-\mathrm{C} 1-\mathrm{C} 10-\mathrm{C} 11$ & $-82.13(18)$ \\
\hline $\mathrm{C} 13 \mathrm{~A}-\mathrm{C} 1 \mathrm{~A}-\mathrm{C} 10 \mathrm{~A}-\mathrm{C} 11 \mathrm{~A}$ & $-31.45(17)$ & $\mathrm{C} 13-\mathrm{C} 1-\mathrm{C} 10-\mathrm{C} 11$ & $33.30(17)$ \\
\hline $\mathrm{O} 12 \mathrm{~A}-\mathrm{C} 11 \mathrm{~A}-\mathrm{C} 10 \mathrm{~A}-\mathrm{C} 9 \mathrm{~A}$ & $158.13(15)$ & $\mathrm{C} 13-\mathrm{O} 12-\mathrm{C} 11-\mathrm{C}^{\prime}$ & $148.84(17)$ \\
\hline $\mathrm{C} 1 \mathrm{~A}^{\prime}-\mathrm{C} 11 \mathrm{~A}-\mathrm{C} 10 \mathrm{~A}-\mathrm{C} 9 \mathrm{~A}$ & $-81.6(2)$ & $\mathrm{C} 13-\mathrm{O} 12-\mathrm{C} 11-\mathrm{C} 10$ & $23.1(2)$ \\
\hline $\mathrm{O} 12 \mathrm{~A}-\mathrm{C} 11 \mathrm{~A}-\mathrm{C} 10 \mathrm{~A}-\mathrm{C} 1 \mathrm{~A}$ & $36.27(17)$ & $\mathrm{C} 9-\mathrm{C} 10-\mathrm{C} 11-\mathrm{O} 12$ & $-156.02(15)$ \\
\hline $\mathrm{C} 1 \mathrm{~A}^{\prime}-\mathrm{C} 11 \mathrm{~A}-\mathrm{C} 10 \mathrm{~A}-\mathrm{C} 1 \mathrm{~A}$ & $156.57(16)$ & $\mathrm{C} 1-\mathrm{C} 10-\mathrm{C} 11-\mathrm{O} 12$ & $-34.98(17)$ \\
\hline $\mathrm{O} 12 \mathrm{~A}-\mathrm{C} 11 \mathrm{~A}-\mathrm{C} 10 \mathrm{~A}-\mathrm{C} 16 \mathrm{~A}$ & $-74.39(18)$ & $\mathrm{C} 16-\mathrm{C} 10-\mathrm{C} 11-\mathrm{O} 12$ & $75.49(19)$ \\
\hline $\mathrm{C} 1 \mathrm{~A}^{\prime}-\mathrm{C} 11 \mathrm{~A}-\mathrm{C} 10 \mathrm{~A}-\mathrm{C} 16 \mathrm{~A}$ & $45.9(2)$ & $\mathrm{C} 9-\mathrm{C} 10-\mathrm{C} 11-\mathrm{C}^{\prime}$ & $84.4(2)$ \\
\hline $\mathrm{C} 2 \mathrm{~A}-\mathrm{C} 1 \mathrm{~A}-\mathrm{N} 14 \mathrm{~A}-\mathrm{C} 15 \mathrm{~A}$ & $168.15(16)$ & $\mathrm{C} 1-\mathrm{C} 10-\mathrm{C} 11-\mathrm{C} 1^{\prime}$ & $-154.55(16)$ \\
\hline $\mathrm{C} 10 \mathrm{~A}-\mathrm{C} 1 \mathrm{~A}-\mathrm{N} 14 \mathrm{~A}-\mathrm{C} 15 \mathrm{~A}$ & $43.74(18)$ & $\mathrm{C} 16-\mathrm{C} 10-\mathrm{C} 11-\mathrm{C}^{\prime}$ & $-44.1(2)$ \\
\hline $\mathrm{C} 13 \mathrm{~A}-\mathrm{C} 1 \mathrm{~A}-\mathrm{N} 14 \mathrm{~A}-\mathrm{C} 15 \mathrm{~A}$ & $-64.6(2)$ & $\mathrm{C} 2-\mathrm{C} 1-\mathrm{N} 14-\mathrm{C} 17$ & $62.7(2)$ \\
\hline $\mathrm{C} 2 \mathrm{~A}-\mathrm{C} 1 \mathrm{~A}-\mathrm{N} 14 \mathrm{~A}-\mathrm{C} 17 \mathrm{~A}$ & $-64.0(2)$ & $\mathrm{C} 10-\mathrm{C} 1-\mathrm{N} 14-\mathrm{C} 17$ & $-172.91(18)$ \\
\hline $\mathrm{C} 10 \mathrm{~A}-\mathrm{C} 1 \mathrm{~A}-\mathrm{N} 14 \mathrm{~A}-\mathrm{C} 17 \mathrm{~A}$ & $171.59(17)$ & $\mathrm{C} 13-\mathrm{C} 1-\mathrm{N} 14-\mathrm{C} 17$ & $-64.3(2)$ \\
\hline $\mathrm{C} 13 \mathrm{~A}-\mathrm{C} 1 \mathrm{~A}-\mathrm{N} 14 \mathrm{~A}-\mathrm{C} 17 \mathrm{~A}$ & $63.2(2)$ & $\mathrm{C} 2-\mathrm{C} 1-\mathrm{N} 14-\mathrm{C} 15$ & $-168.57(16)$ \\
\hline $\mathrm{O} 12 \mathrm{~A}-\mathrm{C} 11 \mathrm{~A}-\mathrm{C} 1 \mathrm{~A}^{\prime}-\mathrm{C} 6 \mathrm{~A}^{\prime}$ & $26.3(3)$ & $\mathrm{C} 10-\mathrm{C} 1-\mathrm{N} 14-\mathrm{C} 15$ & $-44.15(18)$ \\
\hline
\end{tabular}




\begin{tabular}{|c|c|c|c|}
\hline $\mathrm{C} 10 \mathrm{~A}-\mathrm{C} 11 \mathrm{~A}-\mathrm{C} 1 \mathrm{~A}^{\prime}-\mathrm{C} 6 \mathrm{~A}^{\prime}$ & $-90.6(2)$ & $\mathrm{C} 13-\mathrm{C} 1-\mathrm{N} 14-\mathrm{C} 15$ & $64.5(2)$ \\
\hline $\mathrm{O} 12 \mathrm{~A}-\mathrm{C} 11 \mathrm{~A}-\mathrm{C} 1 \mathrm{~A}^{\prime}-\mathrm{C} 2 \mathrm{~A}^{\prime}$ & $-153.38(17)$ & $\mathrm{C} 7-\mathrm{N} 8-\mathrm{C} 9-\mathrm{O} 9$ & $-167.2(2)$ \\
\hline $\mathrm{C} 10 \mathrm{~A}-\mathrm{C} 11 \mathrm{~A}-\mathrm{C} 1 \mathrm{~A}^{\prime}-\mathrm{C} 2 \mathrm{~A}^{\prime}$ & $89.7(2)$ & $\mathrm{C} 7-\mathrm{N} 8-\mathrm{C} 9-\mathrm{C} 10$ & $14.3(3)$ \\
\hline $\mathrm{C} 6 \mathrm{~A}-\mathrm{C} 7 \mathrm{~A}-\mathrm{C} 2 \mathrm{~A}-\mathrm{C} 3 \mathrm{~A}$ & $0.3(3)$ & $\mathrm{C} 1-\mathrm{C} 10-\mathrm{C} 9-\mathrm{O} 9$ & $145.7(2)$ \\
\hline $\mathrm{N} 8 \mathrm{~A}-\mathrm{C} 7 \mathrm{~A}-\mathrm{C} 2 \mathrm{~A}-\mathrm{C} 3 \mathrm{~A}$ & $-177.79(19)$ & $\mathrm{C} 16-\mathrm{C} 10-\mathrm{C} 9-\mathrm{O} 9$ & $30.2(3)$ \\
\hline $\mathrm{C} 6 \mathrm{~A}-\mathrm{C} 7 \mathrm{~A}-\mathrm{C} 2 \mathrm{~A}-\mathrm{C} 1 \mathrm{~A}$ & $178.77(18)$ & $\mathrm{C} 11-\mathrm{C} 10-\mathrm{C} 9-\mathrm{O} 9$ & $-99.9(2)$ \\
\hline $\mathrm{N} 8 \mathrm{~A}-\mathrm{C} 7 \mathrm{~A}-\mathrm{C} 2 \mathrm{~A}-\mathrm{C} 1 \mathrm{~A}$ & $0.6(3)$ & $\mathrm{C} 1-\mathrm{C} 10-\mathrm{C} 9-\mathrm{N} 8$ & $-35.7(2)$ \\
\hline $\mathrm{N} 14 \mathrm{~A}-\mathrm{C} 1 \mathrm{~A}-\mathrm{C} 2 \mathrm{~A}-\mathrm{C} 7 \mathrm{~A}$ & $-99.4(2)$ & $\mathrm{C} 16-\mathrm{C} 10-\mathrm{C} 9-\mathrm{N} 8$ & $-151.28(19)$ \\
\hline $\mathrm{C} 10 \mathrm{~A}-\mathrm{C} 1 \mathrm{~A}-\mathrm{C} 2 \mathrm{~A}-\mathrm{C} 7 \mathrm{~A}$ & $18.2(2)$ & $\mathrm{C} 11-\mathrm{C} 10-\mathrm{C} 9-\mathrm{N} 8$ & $78.7(2)$ \\
\hline $\mathrm{C} 13 \mathrm{~A}-\mathrm{C} 1 \mathrm{~A}-\mathrm{C} 2 \mathrm{~A}-\mathrm{C} 7 \mathrm{~A}$ & $130.44(18)$ & $\mathrm{C} 11-\mathrm{O} 12-\mathrm{C} 13-\mathrm{O} 13$ & $179.06(19)$ \\
\hline $\mathrm{N} 14 \mathrm{~A}-\mathrm{C} 1 \mathrm{~A}-\mathrm{C} 2 \mathrm{~A}-\mathrm{C} 3 \mathrm{~A}$ & $78.9(2)$ & $\mathrm{C} 11-\mathrm{O} 12-\mathrm{C} 13-\mathrm{C} 1$ & $-1.1(2)$ \\
\hline $\mathrm{C} 10 \mathrm{~A}-\mathrm{C} 1 \mathrm{~A}-\mathrm{C} 2 \mathrm{~A}-\mathrm{C} 3 \mathrm{~A}$ & $-163.43(18)$ & $\mathrm{N} 14-\mathrm{C} 1-\mathrm{C} 13-\mathrm{O} 13$ & $48.4(3)$ \\
\hline $\mathrm{C} 13 \mathrm{~A}-\mathrm{C} 1 \mathrm{~A}-\mathrm{C} 2 \mathrm{~A}-\mathrm{C} 3 \mathrm{~A}$ & $-51.2(2)$ & $\mathrm{C} 2-\mathrm{C} 1-\mathrm{C} 13-\mathrm{O} 13$ & $-81.7(3)$ \\
\hline $\mathrm{C} 9 \mathrm{~A}-\mathrm{C} 10 \mathrm{~A}-\mathrm{C} 16 \mathrm{~A}-\mathrm{C} 15 \mathrm{~A}$ & $-114.59(18)$ & $\mathrm{C} 10-\mathrm{C} 1-\mathrm{C} 13-\mathrm{O} 13$ & $158.5(2)$ \\
\hline $\mathrm{C} 1 \mathrm{~A}-\mathrm{C} 10 \mathrm{~A}-\mathrm{C} 16 \mathrm{~A}-\mathrm{C} 15 \mathrm{~A}$ & $8.91(19)$ & $\mathrm{N} 14-\mathrm{C} 1-\mathrm{C} 13-\mathrm{O} 12$ & $-131.46(18)$ \\
\hline $\mathrm{C} 11 \mathrm{~A}-\mathrm{C} 10 \mathrm{~A}-\mathrm{C} 16 \mathrm{~A}-\mathrm{C} 15 \mathrm{~A}$ & $118.69(17)$ & $\mathrm{C} 2-\mathrm{C} 1-\mathrm{C} 13-\mathrm{O} 12$ & $98.43(18)$ \\
\hline $\mathrm{C} 11 \mathrm{~A}-\mathrm{O} 12 \mathrm{~A}-\mathrm{C} 13 \mathrm{~A}-\mathrm{O} 13 \mathrm{~A}$ & $-174.39(19)$ & $\mathrm{C} 10-\mathrm{C} 1-\mathrm{C} 13-\mathrm{O} 12$ & $-21.30(19)$ \\
\hline $\mathrm{C} 11 \mathrm{~A}-\mathrm{O} 12 \mathrm{~A}-\mathrm{C} 13 \mathrm{~A}-\mathrm{C} 1 \mathrm{~A}$ & $6.6(2)$ & $\mathrm{O} 12-\mathrm{C} 11-\mathrm{C} 1^{\prime}-\mathrm{C}^{\prime}$ & $-19.7(3)$ \\
\hline $\mathrm{N} 14 \mathrm{~A}-\mathrm{C} 1 \mathrm{~A}-\mathrm{C} 13 \mathrm{~A}-\mathrm{O} 13 \mathrm{~A}$ & $-52.4(3)$ & $\mathrm{C} 10-\mathrm{C} 11-\mathrm{C} 1^{\prime}-\mathrm{C}^{\prime}$ & $96.8(3)$ \\
\hline $\mathrm{C} 2 \mathrm{~A}-\mathrm{C} 1 \mathrm{~A}-\mathrm{C} 13 \mathrm{~A}-\mathrm{O} 13 \mathrm{~A}$ & $76.9(3)$ & $\mathrm{O} 12-\mathrm{C} 11-\mathrm{C} 1^{\prime}-\mathrm{C} 2^{\prime}$ & $159.99(18)$ \\
\hline $\mathrm{C} 10 \mathrm{~A}-\mathrm{C} 1 \mathrm{~A}-\mathrm{C} 13 \mathrm{~A}-\mathrm{O} 13 \mathrm{~A}$ & $-162.0(2)$ & $\mathrm{C} 10-\mathrm{C} 11-\mathrm{C} 1^{\prime}-\mathrm{C} 2^{\prime}$ & $-83.5(2)$ \\
\hline $\mathrm{N} 14 \mathrm{~A}-\mathrm{C} 1 \mathrm{~A}-\mathrm{C} 13 \mathrm{~A}-\mathrm{O} 12 \mathrm{~A}$ & $126.50(17)$ & $\mathrm{C} 7-\mathrm{C} 2-\mathrm{C} 3-\mathrm{C} 4$ & $-1.5(3)$ \\
\hline $\mathrm{C} 2 \mathrm{~A}-\mathrm{C} 1 \mathrm{~A}-\mathrm{C} 13 \mathrm{~A}-\mathrm{O} 12 \mathrm{~A}$ & $-104.16(18)$ & $\mathrm{C} 1-\mathrm{C} 2-\mathrm{C} 3-\mathrm{C} 4$ & $179.28(19)$ \\
\hline $\mathrm{C} 10 \mathrm{~A}-\mathrm{C} 1 \mathrm{~A}-\mathrm{C} 13 \mathrm{~A}-\mathrm{O} 12 \mathrm{~A}$ & $16.9(2)$ & $\mathrm{C} 2-\mathrm{C} 3-\mathrm{C} 4-\mathrm{C} 5$ & $1.9(3)$ \\
\hline $\mathrm{C} 8 \mathrm{~A}^{\prime}-\mathrm{O} 7 \mathrm{~A}^{\prime}-\mathrm{C} 2 \mathrm{~A}^{\prime}-\mathrm{C}^{\prime} \mathrm{A}^{\prime}$ & $-146.66(19)$ & $\mathrm{C} 6^{\prime}-\mathrm{C} 1^{\prime}-\mathrm{C} 2^{\prime}-\mathrm{O} 7^{\prime}$ & $175.2(3)$ \\
\hline $\mathrm{C} 8 \mathrm{~A}^{\prime}-07 \mathrm{~A}^{\prime}-\mathrm{C} 2 \mathrm{~A}^{\prime}-\mathrm{C} 3 \mathrm{~A}^{\prime}$ & $39.6(3)$ & $\mathrm{C} 11-\mathrm{C} 1^{\prime}-\mathrm{C}^{\prime}-\mathrm{O}^{\prime}$ & $-4.5(3)$ \\
\hline $\mathrm{C}^{2} \mathrm{~A}^{\prime}-\mathrm{C} 1 \mathrm{~A}^{\prime}-\mathrm{C} 2 \mathrm{~A}^{\prime}-\mathrm{O} 7 \mathrm{~A}^{\prime}$ & $-175.19(19)$ & $\mathrm{C} 6^{\prime}-\mathrm{C} 1^{\prime}-\mathrm{C} 2^{\prime}-\mathrm{C} 3^{\prime}$ & $-1.2(4)$ \\
\hline $\mathrm{C} 11 \mathrm{~A}-\mathrm{C} 1 \mathrm{~A}^{\prime}-\mathrm{C} 2 \mathrm{~A}^{\prime}-\mathrm{O} 7 \mathrm{~A}^{\prime}$ & $4.5(3)$ & $\mathrm{C} 11-\mathrm{C} 1^{\prime}-\mathrm{C} 2^{\prime}-\mathrm{C} 3^{\prime}$ & $179.14(19)$ \\
\hline $\mathrm{C} 6 \mathrm{~A}^{\prime}-\mathrm{C} 1 \mathrm{~A}^{\prime}-\mathrm{C} 2 \mathrm{~A}^{\prime}-\mathrm{C} 3 \mathrm{~A}^{\prime}$ & $-1.1(3)$ & $\mathrm{C} 9-\mathrm{C} 10-\mathrm{C} 16-\mathrm{C} 15$ & $109.63(19)$ \\
\hline $\mathrm{C} 11 \mathrm{~A}-\mathrm{C} 1 \mathrm{~A}^{\prime}-\mathrm{C} 2 \mathrm{~A}^{\prime}-\mathrm{C} 3 \mathrm{~A}^{\prime}$ & $178.60(18)$ & $\mathrm{C} 1-\mathrm{C} 10-\mathrm{C} 16-\mathrm{C} 15$ & $-12.0(2)$ \\
\hline $\mathrm{O}^{2} \mathrm{~A}^{\prime}-\mathrm{C} 2 \mathrm{~A}^{\prime}-\mathrm{C} 3 \mathrm{~A}^{\prime}-\mathrm{O} 9 \mathrm{~A}^{\prime}$ & $-2.0(3)$ & $\mathrm{C} 11-\mathrm{C} 10-\mathrm{C} 16-\mathrm{C} 15$ & $-121.91(18)$ \\
\hline $\mathrm{C} 1 \mathrm{~A}^{\prime}-\mathrm{C} 2 \mathrm{~A}^{\prime}-\mathrm{C} 3 \mathrm{~A}^{\prime}-09 \mathrm{~A}^{\prime}$ & $-175.4(2)$ & $\mathrm{C} 2-\mathrm{C} 7-\mathrm{C} 6-\mathrm{C} 5$ & $0.9(3)$ \\
\hline $\mathrm{O}^{7} \mathrm{~A}^{\prime}-\mathrm{C} 2 \mathrm{~A}^{\prime}-\mathrm{C} 3 \mathrm{~A}^{\prime}-\mathrm{C}^{\prime} \mathrm{A}^{\prime}$ & $175.2(2)$ & $\mathrm{N} 8-\mathrm{C} 7-\mathrm{C} 6-\mathrm{C} 5$ & $-177.4(2)$ \\
\hline $\mathrm{C} 1 \mathrm{~A}^{\prime}-\mathrm{C} 2 \mathrm{~A}^{\prime}-\mathrm{C} 3 \mathrm{~A}^{\prime}-\mathrm{C} 4 \mathrm{~A}^{\prime}$ & $1.7(3)$ & $\mathrm{C} 7-\mathrm{C} 6-\mathrm{C} 5-\mathrm{C} 4$ & $-0.5(3)$ \\
\hline $\mathrm{C} 7 \mathrm{~A}-\mathrm{C} 2 \mathrm{~A}-\mathrm{C} 3 \mathrm{~A}-\mathrm{C} 4 \mathrm{~A}$ & $1.5(3)$ & $\mathrm{C} 3-\mathrm{C} 4-\mathrm{C} 5-\mathrm{C} 6$ & $-0.8(3)$ \\
\hline $\mathrm{C} 1 \mathrm{~A}-\mathrm{C} 2 \mathrm{~A}-\mathrm{C} 3 \mathrm{~A}-\mathrm{C} 4 \mathrm{~A}$ & $-176.8(2)$ & $\mathrm{C} 1^{\prime}-\mathrm{C} 2^{\prime}-\mathrm{O} 7^{\prime}-\mathrm{C} 8^{\prime}$ & $164.7(3)$ \\
\hline $\mathrm{C} 17 \mathrm{~A}-\mathrm{N} 14 \mathrm{~A}-\mathrm{C} 15 \mathrm{~A}-\mathrm{C} 16 \mathrm{~A}$ & $-169.27(17)$ & $\mathrm{C} 3^{\prime}-\mathrm{C} 2^{\prime}-\mathrm{O} 7^{\prime}-\mathrm{C} 8^{\prime}$ & $-19.2(4)$ \\
\hline $\mathrm{C} 1 \mathrm{~A}-\mathrm{N} 14 \mathrm{~A}-\mathrm{C} 15 \mathrm{~A}-\mathrm{C} 16 \mathrm{~A}$ & $-38.11(19)$ & $\mathrm{C} 1-\mathrm{N} 14-\mathrm{C} 15-\mathrm{C} 16$ & $36.4(2)$ \\
\hline $\mathrm{C} 10 \mathrm{~A}-\mathrm{C} 16 \mathrm{~A}-\mathrm{C} 15 \mathrm{~A}-\mathrm{N} 14 \mathrm{~A}$ & $16.9(2)$ & $\mathrm{C} 17-\mathrm{N} 14-\mathrm{C} 15-\mathrm{C} 16$ & $167.98(18)$ \\
\hline $\mathrm{C} 2 \mathrm{~A}-\mathrm{C} 7 \mathrm{~A}-\mathrm{C} 6 \mathrm{~A}-\mathrm{C} 5 \mathrm{~A}$ & $-1.4(3)$ & $\mathrm{C} 10-\mathrm{C} 16-\mathrm{C} 15-\mathrm{N} 14$ & $-14.0(2)$ \\
\hline $\mathrm{N} 8 \mathrm{~A}-\mathrm{C} 7 \mathrm{~A}-\mathrm{C} 6 \mathrm{~A}-\mathrm{C} 5 \mathrm{~A}$ & $176.7(2)$ & $\mathrm{O} 7^{\prime}-\mathrm{C} 2^{\prime}-\mathrm{C} 3^{\prime}-\mathrm{O}^{\prime}$ & $6.2(4)$ \\
\hline $\mathrm{C} 4 \mathrm{~A}^{\prime}-\mathrm{C} 3 \mathrm{~A}^{\prime}-\mathrm{O} 9 \mathrm{~A}^{\prime}-\mathrm{C} 10 \mathrm{~B}$ & $24.3(4)$ & $\mathrm{C} 1^{\prime}-\mathrm{C} 2^{\prime}-\mathrm{C} 3^{\prime}-\mathrm{O} 9^{\prime}$ & $-177.9(2)$ \\
\hline $\mathrm{C} 2 \mathrm{~A}^{\prime}-\mathrm{C} 3 \mathrm{~A}^{\prime}-\mathrm{O} 9 \mathrm{~A}^{\prime}-\mathrm{C} 10 \mathrm{~B}$ & $-158.7(2)$ & $\mathrm{O} 7^{\prime}-\mathrm{C} 2^{\prime}-\mathrm{C} 3^{\prime}-\mathrm{C} 4^{\prime}$ & $-174.0(3)$ \\
\hline $\mathrm{C} 2 \mathrm{~A}^{\prime}-\mathrm{C} 1 \mathrm{~A}^{\prime}-\mathrm{C} 6 \mathrm{~A}^{\prime}-\mathrm{C} 5 \mathrm{~A}^{\prime}$ & $-0.1(4)$ & $\mathrm{C} 1^{\prime}-\mathrm{C} 2^{\prime}-\mathrm{C} 3^{\prime}-\mathrm{C} 4^{\prime}$ & $1.9(4)$ \\
\hline $\mathrm{C} 11 \mathrm{~A}-\mathrm{C} 1 \mathrm{~A}^{\prime}-\mathrm{C} 6 \mathrm{~A}^{\prime}-\mathrm{C} 5 \mathrm{~A}^{\prime}$ & $-179.8(2)$ & $\mathrm{C} 2^{\prime}-\mathrm{C} 1^{\prime}-\mathrm{C} 6^{\prime}-\mathrm{C} 5^{\prime}$ & $0.0(5)$ \\
\hline $\mathrm{O} 9 \mathrm{~A}^{\prime}-\mathrm{C} 3 \mathrm{~A}^{\prime}-\mathrm{C} 4 \mathrm{~A}^{\prime}-\mathrm{C} 5 \mathrm{~A}^{\prime}$ & $175.9(3)$ & $\mathrm{C} 11-\mathrm{C} 1^{\prime}-\mathrm{C} 6^{\prime}-\mathrm{C} 5^{\prime}$ & $179.6(3)$ \\
\hline $\mathrm{C} 2 \mathrm{~A}^{\prime}-\mathrm{C} 3 \mathrm{~A}^{\prime}-\mathrm{C} 4 \mathrm{~A}^{\prime}-\mathrm{C} 5 \mathrm{~A}^{\prime}$ & $-1.1(4)$ & $\mathrm{C} 4^{\prime}-\mathrm{C} 3^{\prime}-\mathrm{O}^{\prime}-\mathrm{C} 10^{\prime}$ & $-23.3(4)$ \\
\hline $\mathrm{C} 2 \mathrm{~A}-\mathrm{C} 3 \mathrm{~A}-\mathrm{C} 4 \mathrm{~A}-\mathrm{C} 5 \mathrm{~A}$ & $-2.3(4)$ & $\mathrm{C} 2^{\prime}-\mathrm{C} 3^{\prime}-\mathrm{O}^{\prime}-\mathrm{C} 10^{\prime}$ & $156.6(3)$ \\
\hline
\end{tabular}




\begin{tabular}{llll}
$\mathrm{C} 3 \mathrm{~A}-\mathrm{C} 4 \mathrm{~A}-\mathrm{C} 5 \mathrm{~A}-\mathrm{C} 6 \mathrm{~A}$ & $1.2(4)$ & $\mathrm{C} 1^{\prime}-\mathrm{C} 6^{\prime}-\mathrm{C} 5^{\prime}-\mathrm{C} 4^{\prime}$ & $0.6(6)$ \\
$\mathrm{C} 7 \mathrm{~A}-\mathrm{C} 6 \mathrm{~A}-\mathrm{C} 5 \mathrm{~A}-\mathrm{C} 4 \mathrm{~A}$ & $0.6(4)$ & $\mathrm{O} 9^{\prime}-\mathrm{C} 3^{\prime}-\mathrm{C} 4^{\prime}-\mathrm{C} 5^{\prime}$ & $178.5(3)$ \\
$\mathrm{C} 1 \mathrm{~A}^{\prime}-\mathrm{C} 6 \mathrm{~A}^{\prime}-\mathrm{C} 5 \mathrm{~A}^{\prime}-\mathrm{C} 4 \mathrm{~A}^{\prime}$ & $0.7(4)$ & $\mathrm{C} 2^{\prime}-\mathrm{C} 3^{\prime}-\mathrm{C} 4^{\prime}-\mathrm{C} 5^{\prime}$ & $-1.4(5)$ \\
$\mathrm{C} 3 \mathrm{~A}^{\prime}-\mathrm{C} 4 \mathrm{~A}^{\prime}-\mathrm{C} 5 \mathrm{~A}^{\prime}-\mathrm{C} 6 \mathrm{~A}^{\prime}$ & $-0.1(4)$ & $\mathrm{C} 6^{\prime}-\mathrm{C} 5^{\prime}-\mathrm{C} 4^{\prime}-\mathrm{C} 3^{\prime}$ & $0.1(6)$ \\
\hline
\end{tabular}

Hydrogen-bond geometry $\left(A,{ }^{\circ}\right)$

\begin{tabular}{lllll}
\hline$D-\mathrm{H} \cdots A$ & $D-\mathrm{H}$ & $\mathrm{H} \cdots A$ & $D \cdots A$ & $D-\mathrm{H} \cdots A$ \\
\hline $\mathrm{N} 8-\mathrm{H} 8 \cdots \mathrm{O}^{\mathrm{i}}$ & $0.89(3)$ & $2.01(3)$ & $2.903(2)$ & $177(2)$ \\
$\mathrm{N} 8 A-\mathrm{H} 8 A^{\cdots} \cdots \mathrm{O} 9 A^{\mathrm{ii}}$ & $0.89(2)$ & $2.07(3)$ & $2.958(2)$ & $175(2)$ \\
$\mathrm{C} 6 A^{\prime}-\mathrm{H} 6 A^{\prime} \cdots \mathrm{O} 13 A^{\mathrm{iii}}$ & 0.93 & 2.43 & $3.322(3)$ & 161
\end{tabular}

Symmetry codes: (i) $-x,-y+1,-z+1$; (ii) $-x+1,-y+1,-z$; (iii) $-x+2,-y,-z$. 